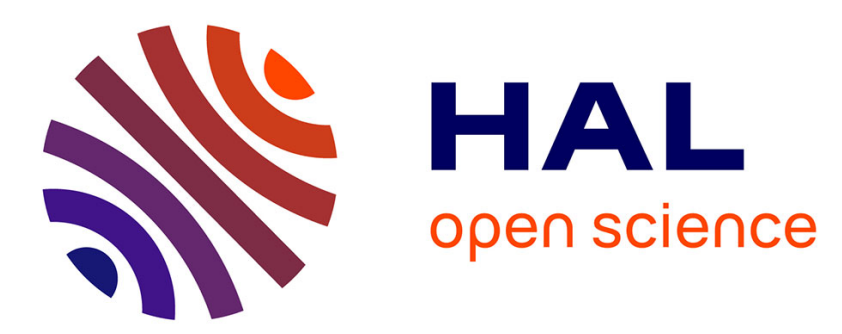

\title{
Model-based modes detection and discernibility for switched affine discrete-time systems
}

\author{
Meriem Halimi, Gilles Millérioux, Jamal Daafouz
}

\section{To cite this version:}

Meriem Halimi, Gilles Millérioux, Jamal Daafouz. Model-based modes detection and discernibility for switched affine discrete-time systems. IEEE Transactions on Automatic Control, 2015, 60 (6), pp.1501-1514. 10.1109/TAC.2014.2383012 . hal-01185926

\author{
HAL Id: hal-01185926 \\ https://hal.science/hal-01185926
}

Submitted on 25 Sep 2015

HAL is a multi-disciplinary open access archive for the deposit and dissemination of scientific research documents, whether they are published or not. The documents may come from teaching and research institutions in France or abroad, or from public or private research centers.
L'archive ouverte pluridisciplinaire HAL, est destinée au dépôt et à la diffusion de documents scientifiques de niveau recherche, publiés ou non, émanant des établissements d'enseignement et de recherche français ou étrangers, des laboratoires publics ou privés. 


\title{
A tutorial on model-based modes detection and discernibility for switched affine discrete-time systems
}

\author{
Meriem Halimi*, Gilles Millerioux*, Jamal Daafouz*
}

\begin{abstract}
This paper addresses the problem of modes detection for switching discrete-time linear and affine systems. A unified presentation of the model-based methods with the corresponding modes detectors is carried out and a comparative study is conducted. Next, the different notions of discernibility proposed in the literature are presented. Such notions are central since they guarantee the uniqueness of the solution delivered by the modes detectors. A complete step-by-step procedure of modes detection is proposed. The paper includes, whenever necessary, some extensions of the existing results.
\end{abstract}

\section{INTRODUCTION}

Systems with continuous and discrete dynamics which interplay one another are known under the name "hybrid systems". Over the last two decades, particular attention has been devoted to those systems. Among several subclasses of hybrid systems, switching linear systems are of special importance. They are linear systems for which the state space realization matrices can take values in a finite set of indexed matrices. The index corresponding to the active linear system is called the "mode" or the "discrete state". The way how the index evolves in time is orchestrated by a socalled switching rule. Many purposes require the availability of the modes like observer synthesis, identification, gain scheduling state feedback control, fault diagnosis. Typical real-worl applications have been addressed, for example, in [1] (pick-and-place machine), in [2] (greenhouse), in [3] (circuits with electronic switches) to mention a few. When the mode is known, efficient approaches for achieving observer or controller synthesis have been proposed in [4] [5] [6]. The design consists in constructing obervers gains for each individual mode and the global convergence is ensured by resorting to a common Lyapunov function. Observer design approches have been proposed in the case when the mode is unknown (see [7] for example) but with more limited performances or under more restrictive assumptions (global convergence no longer guaranteed, limited number of modes, ...). Unfortunately, the situation where the modes are not accessible is not scarce. Two typical situations may occur in this respect. The switch may be triggered by an unknown external event or the switch may depend on a non accessible internal state on which depends the switching rule. Let us note that different terminologies with a somewhat similar meaning are used in the literature: modes reconstruction, modes detection or modes estimation.

The problem of modes detection for switching systems has received a lot of attention. We can split the modes detection methods into two different classes: the data-based approaches and the modelbased approaches. The data-based approaches are most often based on batches of data and the modes detection is jointly performed with the identification of the parameters of each local model. The main data-based methods are algebraic approaches [8], clustering approaches [9], bounded-error approaches [10], sparsity and convex relaxation approaches [11] and time order-based approaches [12]. All those methods are in general not really suitable for on-line applications like output-feedback control or gain scheduling control. However, some of them could be adapted and they are essential if

$\begin{array}{cccccc}* \text { Research } & \begin{array}{c}\text { Center } \\ \text { (CRAN), for }\end{array} \text { Université } & \text { Automatic } & \text { Control of } & \text { Nancy } \\ \text { Lorraine, } & \text { Corresponding } & \text { author }\end{array}$

the model parameters for each mode are unknown. Indeed, modelbased approaches assume that each local model is known. A class of model-based methods also considers batches of input/output data and the modes detection cannot be performed on-line. We can mention the approach proposed in [13] whose principle relies on the consideration of the dimension of projected subspaces. It is a refinement of other similar subspaces framework as proposed in [14], [15]. Other model-based modes detections methodologies call for filters-based techniques. Filters aim at both reconstructing the continuous state and the modes. Some methods are based on a bank of observers whose convergence is conditioned by a minimum dwell time of the mode causing a delay in the detection [16] [17]. They are also called failures detection when they are used in diagnosis [18] [19]. To relax the asymptotical behavior of such detectors, modelbased approaches with moving-horizon, also called receding horizon, strategies have been suggested. Receding horizon techniques use a limited amount of more recent information for the estimation. A joint continuous state and modes estimation has been proposed in [20]. It consists in minimizing a criterion involving both the estimated quantities, namely the continuous and the discrete state, and the output data in a moving finite time interval of time. More recently, a technique using a special class of particle filters has been proposed in [21] but is devoted to autonomous nonlinear systems. On the fringe of the pure scope of the present work because it is restricted to continous time systems, let us mention the differentiation technique presented in [22]. Finally, the works [13] [23] [24] are exclusively concerned with modes estimation, independently from the continuous state estimation. In the literature devoted to diagnosis, they are called, parity space approaches and are suitable for on-line applications.

An important concept for modes detection purpose is the so-called discernibility also called in some papers distinguishability. Roughly speaking, discernibility reflects the ability for a modes detector of discriminating the active sequence from any other ones. In other words, it deals with the matter of uniqueness of the solution delivered by the detector. A sufficient condition for discernibility to hold is the so-called mode observability as introduced in [25]. Such a notion has also been investigated in [26]-[28] and revisited to be appropriate to nonlinear switched systems or to cope with noisy measurements. Less restrictive conditions can be found in the literature like Backward Discernibility and Forward Discernibility [23], $(\eta, \omega)$-discernibility [20]. For controlled systems, discernibility deserves a special treatment because it depends on the input sequence. In this respect, the so-called active mode observation has been addressed in [29], [30] and [31] where a distinction between discerning control sequences over a finite or an infinite horizon of time is made.

This paper aims at presenting, in a tutorial-like form and in a unified way, the main model-based modes detection methods dedicated to switched linear (and by extension affine) discrete-time systems, together with the concept of discernibility. Many different notions and results have been presented over the literature. However, they are not unified and a complete modes detection procedure involving all those complementary notions is really lacking. The present work includes, whenever necessary, some new results although some of them are quite straightforward but should however be considered as useful for the sake of unification. 
The outline is as follows. In Section I, a unified presentation of the main model-based modes detection methods, which apply for switched linear and affine systems, with the corresponding modes detector structures is carried out. In Section II, a comparative study is conducted. The problem of uniqueness of the modes detection is discussed in Section III through the basic notion of discernibility. Section IV is devoted to advanced notions of discernibility and the corresponding procedures for modes detection. Section V deals with Backward Discernibility, a concept which allows to reduce the computational cost of the modes detection. A complete step-by-step procedure involving in a coherent way all the results is proposed in Section VI. An illustrative example is proposed in Section VII to clarify the main points.

Notation: $\mathbb{N}$ is the set of natural numbers. $\mathbb{R}^{n}$ is the $n$-dimensional set of real numbers. $\mathbf{1}$ is the identity matrix and $\mathbf{0}$ is the zero matrix, being both of appropriate dimension when unspecified. $X^{T}$ is the transpose of the matrix $X$. The matrix $X^{\dagger}$ stands for the generalized inverse (Moore-Penrose) of $X$ satisfying $X^{\dagger} X$ symmetric, $X X^{\dagger}$ symmetric, $X X^{\dagger} X=X$ and $X^{\dagger} X X^{\dagger}=X^{\dagger}$. The kernel (left null space) of $X$ is denoted $\operatorname{ker}(X) . \mathcal{R}(X)$ is the column range space of $X$. The rank of a matrix $X$ is denoted $\operatorname{rank}(X)$.

\section{Model-BASED MOdes Detection METHOdologies}

\section{A. Preliminaries and problem statement}

We consider the class of switched affine systems given by

$$
\left\{\begin{aligned}
x_{k+1} & =A_{\sigma(k)} x_{k}+B_{\sigma(k)} u_{k}+E_{\sigma(k)} \\
y_{k} & =C_{\sigma(k)} x_{k}+D_{\sigma(k)} u_{k}
\end{aligned}\right.
$$

where $k \in \mathbb{N}$ is the natural number standing for the discretetime, $x_{k} \in \mathbb{R}^{n}$ is the state vector, $y_{k} \in \mathbb{R}^{m}$ is the output, $u_{k} \in \mathbb{R}^{p}$ is the input. The function $\sigma$ is the switching law defined by $\sigma: \mathbb{N} \rightarrow \mathcal{J}=\{1, \ldots, J\}$ that assigns, to any discrete-time $k \in \mathbb{N}$, the integer $\sigma(k) \in \mathcal{J}$. The matrices $A_{\sigma(k)} \in \mathbb{R}^{n \times n}, B_{\sigma(k)} \in \mathbb{R}^{n \times p}$, $C_{\sigma(k)} \in \mathbb{R}^{m \times n}, D_{\sigma(k)} \in \mathbb{R}^{m \times p}$ and $E_{\sigma(k)} \in \mathbb{R}^{n}$ are the state space matrices of the system which belong to the respective finite sets $\left\{A_{1}, \ldots, A_{J}\right\},\left\{B_{1}, \ldots, B_{J}\right\},\left\{C_{1}, \ldots, C_{J}\right\},\left\{D_{1}, \ldots, D_{J}\right\}$ and $\left\{E_{1}, \ldots, E_{J}\right\}$. Hereafter, $\sigma_{\left[k_{1}, k_{2}\right]}$ will stand for the finite sequence of modes (also called path) $\left\{\sigma\left(k_{1}\right), \cdots, \sigma\left(k_{2}\right)\right\}$ in the interval of time $\left[k_{1}, k_{2}\right]=\left[k_{1}, k_{1}+1, \ldots, k_{2}-1, k_{2}\right]$. The active sequence will be denoted with $\sigma^{*}$. Stacking up the successive outputs $y_{k}$ in the finite interval of time $[k-h, k]$ yields

$$
y_{k-h, k}=\mathcal{O}_{\sigma_{[k-h, k]}} x_{k-h}+T_{\sigma_{[k-h, k]}} u_{k-h, k}+T_{\sigma_{[k-h, k]}^{\prime}}^{\prime}
$$

where

$$
\mathcal{O}_{\sigma_{[k-h, k]}}=\left[\begin{array}{c}
C_{\sigma(k-h)} \\
C_{\sigma(k-h+1)} A_{\sigma(k-h)} \\
\vdots \\
C_{\sigma(k-1)} A_{\sigma(k-2)}^{\sigma(k-h)} \\
C_{\sigma(k)} A_{\sigma(k-1)}^{\sigma(k-h)}
\end{array}\right]
$$

with $\mathcal{O}_{\sigma_{[k, k]}}=C_{\sigma(k)}$ and $A_{k_{1}}^{k_{2}}=A_{k_{1}} A_{k_{1}+1} \cdots A_{k_{2}}$ for $k_{2} \geq k_{1}$. We recursively define for $h>1$,

$$
T_{\sigma_{[k-h, k]}}=\left[\begin{array}{cc}
D_{\sigma(k-h)} & \mathbf{0} \\
\mathcal{O}_{\sigma_{[k-h+1, k]}} B_{\sigma(k-h)} & T_{\sigma_{[k-h+1, k]}}
\end{array}\right]
$$

with $T_{\sigma_{[k, k]}}=D_{\sigma(k)}$. Finally, we define for $h>0$,

$$
T_{\sigma_{[k-h, k]}}^{\prime}=\left[\begin{array}{c}
\mathbf{0} \\
\mathcal{O}_{\sigma_{[k-h+1, k]}} E_{\sigma(k-h)}
\end{array}\right]
$$

with $T_{\sigma_{[k, k]}}^{\prime}=\mathbf{0}$.

$$
y_{k_{1}, k_{2}}=\left[\begin{array}{c}
y_{k_{1}} \\
y_{k_{1}+1} \\
\vdots \\
y_{k_{2}}
\end{array}\right] \quad, \quad u_{k_{1}, k_{2}}=\left[\begin{array}{c}
u_{k_{1}} \\
u_{k_{1}+1} \\
\vdots \\
u_{k_{2}}
\end{array}\right]
$$

$\mathcal{O}_{\sigma_{[k-h, k]}}$ is the observability matrix in the finite observation window $[k-h, k]$. We denote with $\mathcal{S}$ the set of integers $s \in \mathcal{S}$ that uniquely identify a sequence $\sigma_{s} \in \mathcal{J}^{h+1}$ in the interval of time $[k-h, k]$ and with $S \leq J^{h+1}$ the cardinality of $\mathcal{S}$ which corresponds to the number of admissible sequences in $\mathcal{J}^{h+1}$. By admissible sequence, it is meant a sequence for which $\sigma(k+1)$ is consistent with $\sigma(k)$ and the transition rule defined by the switching function $\sigma$.

Being known sequences of input/output data $u_{k}$ and $y_{k}$ over a prescribed finite length horizon, the aim of the modes detection is to estimate $\sigma(k)$. The estimated mode selected from the active modes sequence will be denoted $\sigma^{*}(k)$. The following subsection is devoted to a unified presentation of model-based approaches that are exclusively dedicated to modes detection.

\section{B. Modes detections}

Owing to their structure inherent to the discrete-time aspect, it turns out that the detectors do not exclusively deliver the active mode $\sigma^{*}(k)$ at time $k$ but deliver the whole or a part of the active sequence $\sigma^{*}$ in the interval of time $[k-h, k]$. The determination of an adequate value for $h$, the way how $\sigma^{*}(k)$ can be derived from the active sequence will be addressed later in this paper. Furthermore, the uniqueness in the modes recovery, that is the property of discernibility, depends on the way how the active mode is inferred from the active sequences delivered by the modes detectors. As a result, discernibility will be discussed after the following comparison of the modes detectors.

1) Detection 1: This corresponds to the modes detection of [23]. The detector is based on the fact that the active sequence $\sigma^{*}$ fulfills

$$
\sigma^{*} \in\left\{\sigma_{s} \in \mathcal{J}^{h+1} \mid y_{k-h, k}-T_{\sigma_{s}} u_{k-h, k}-T_{\sigma_{s}}^{\prime} \in \mathcal{R}\left(\mathcal{O}_{\sigma_{s}}\right)\right\}
$$

where $\mathcal{R}\left(\mathcal{O}_{\sigma_{s}}\right)$ stands for the "column range space" of $\mathcal{O}_{\sigma_{s}}$. The following lemma will be useful for the sequel.

Lemma 1. [23] Given a vector $Y^{\prime}$ and a matrix $\mathcal{O}$, being $X$ unknown, it holds that

$$
Y^{\prime} \in \mathcal{R}(\mathcal{O}) \Leftrightarrow \exists X \mid Y^{\prime}=\mathcal{O} X \Leftrightarrow\left(\mathcal{O O}^{\dagger}-\mathbf{I}\right) Y^{\prime}=\mathbf{0}
$$

Let us define a quantity, called residual

$$
r_{h, \sigma_{s}}^{1}=\left(\mathcal{O}_{\sigma_{s}} \mathcal{O}_{\sigma_{s}}^{\dagger}-\mathbf{1}\right)\left(y_{k-h, k}-T_{\sigma_{s}} u_{k-h, k}-T_{\sigma_{s}}^{\prime}\right)
$$

Then, we have the following proposition.

Proposition 1. The active sequence $\sigma^{*}$ in the interval of time $[k-h, k]$ is a sequence $\sigma_{s} \in \mathcal{J}^{h+1}(s \in \mathcal{S})$ such that the corresponding residual fulfills

$$
r_{h, \sigma_{s}}^{1}=\mathbf{0}
$$

Proof. It is a consequence of Lemma 1, letting $Y^{\prime}=y_{k-h, k}-$ $T_{\sigma_{s}} u_{k-h, k}-T_{\sigma_{s}}^{\prime}$ and $\mathcal{O}=\mathcal{O}_{\sigma_{s}}$ in (7) together with the consideration of (6).

2) Detection 2: This corresponds to the modes detection of [24] where it is assumed the so-called pathwise observability (PWO) [32].

Definition 1. [32] The system (1) is PathWise Observable if there exists an integer $h$ such that every path $\sigma_{s} \in \mathcal{J}^{h+1}(s \in \mathcal{S})$ of length $h+1$ is observable, i.e. satisfies $\operatorname{rank}\left(\mathcal{O}_{\sigma_{s}}\right)=n$. The smallest integer $h$ is called the index of PathWise Observability. 
Assuming that system (1) is PathWise Observable, there exists a non zero and so-called projection matrix fulfilling

$$
\Omega_{\sigma_{s}} \mathcal{O}_{\sigma_{s}}=\mathbf{0}
$$

The matrix $\Omega_{\sigma_{s}}$ corresponds to the left null space of $\mathcal{O}_{\sigma_{s}}$. Introducing the residual defined as

$$
r_{h, \sigma_{s}}^{2}=\Omega_{\sigma_{s}}\left(y_{k-h, k}-T_{\sigma_{s}} u_{k-h, k}-T_{\sigma_{s}}^{\prime}\right)
$$

and left multiplying (2) by $\Omega_{\sigma_{s}}$, when taking into account (10), the following proposition stated in [24] applies.

Proposition 2. [24] The active sequence $\sigma^{*}$ in the interval of time $[k-h, k]$ is a sequence $\sigma_{s} \in \mathcal{J}^{h+1}(s \in \mathcal{S})$ such that the corresponding residual fulfills

$$
r_{h, \sigma_{s}}^{2}=\mathbf{0}
$$

3) Detection 3: Independently of the modes detection problem, a necessary and sufficient condition to get an equivalent I/O (Input/Output) realization, called Switched Auto-Regressive eXogeneous (SARX) representation, of switched affine systems have been proposed in [33]. As it turns out, a new modes detection can be derived from this work. It is explained below. First, let us recall the results of [33] and reformulated here with the notation of the present paper.

Theorem 1. [33] The system (1) admits an equivalent SARX representation if and only if, for every mode sequence $\sigma_{s} \in \mathcal{J}^{h+1}$ $(s \in \mathcal{S})$, there exists an integer $h \in \mathbb{N}$ and a matrix $\Xi_{s}^{(1)}$ verifying

$$
\Xi_{s}^{(1)} \mathcal{O}_{\sigma_{[k-h, k-1]}}=C_{\sigma(k)} A_{\sigma(k-1)}^{\sigma(k-h)}
$$

Corollary 1. [33] Whenever Theorem 1 is fulfilled, the SARX model is given by

$$
y_{k}=\Xi_{s} v_{k}
$$

with

$$
\begin{aligned}
& v_{k}=\left[\begin{array}{lll}
y_{k-h, k-1}^{T} & u_{k-h, k}^{T} & \boldsymbol{I}
\end{array}\right]^{T} \\
& \Xi_{s}=\left[\begin{array}{lll}
\Xi_{s}^{(1)} & \Xi_{s}^{(2)} & \Xi_{s}^{(3)}
\end{array}\right]
\end{aligned}
$$

and where

- $\Xi_{s}^{(1)}$ is the solution of (13),

- $\Xi_{s}^{(2)}=\left[\begin{array}{ll}C_{\sigma(k)} B_{\sigma(k-1)}^{\sigma(k-h)} & D_{\sigma(k)}\end{array}\right]-\Xi_{s}^{(1)} T_{\sigma_{[k-h, k-1]}}$,

- $\Xi_{s}^{(3)}=\left[C_{\sigma(k)} E_{\sigma(k-1)}^{\sigma(k-h)}\right]-\Xi_{s}^{(1)} T_{\sigma_{[k-h, k-1]}^{\prime}}^{\prime}$.

Based on this result, we can derive the following modes detection.

Proposition 3. Whenever Theorem 1 is fulfilled, the active sequence $\sigma^{*}$ in the interval of time $[k-h, k]$ is a sequence $\sigma_{s} \in \mathcal{J}^{h+1}(s \in$ $\mathcal{S})$ such that the corresponding residual fulfills

$$
r_{h, \sigma_{s}}^{3}=\mathbf{0}
$$

with

$$
r_{h, \sigma_{s}}^{3}=\left[\begin{array}{ll}
H_{s} & \boldsymbol{1}
\end{array}\right]\left(y_{k-h, k}-T_{\sigma_{[k-h, k]}} u_{k-h, k}-T_{\sigma_{[k-h, k]}}^{\prime}\right)
$$

where

$$
H_{s}=-\left(C_{\sigma(k)} A_{\sigma(k-1)}^{\sigma(k-h)} \quad \mathcal{O}_{\sigma_{[k-h, k]}}^{\dagger}+Q_{\sigma}^{T}\left(\boldsymbol{1}-\mathcal{O}_{\sigma_{[k-h, k]}} \mathcal{O}_{\sigma_{[k-h, k]}^{\dagger}}^{\dagger}\right)\right)
$$

being $Q_{\sigma}$ an arbitrary matrix.

Proof. Whenever Theorem 1 is fulfilled, according to a classical result of linear algebra, (13) admits an explicit solution that reads

$$
\Xi_{s}^{(1)}=C_{\sigma(k)} A_{\sigma(k-1)}^{\sigma(k-h)} \quad \mathcal{O}_{\sigma_{[k-h, k]}^{\dagger}}^{\dagger}+Q_{\sigma}^{T}\left(\boldsymbol{I}-\mathcal{O}_{\sigma_{[k-h, k]}} \mathcal{O}_{\sigma_{[k-h, k]}^{\dagger}}^{\dagger}\right)
$$

with $Q_{\sigma}$ an arbitrary matrix.

Actually, (19) is a parametrization of all the solutions of (13). Such a solution involves the generalized inverse $\mathcal{O}_{\sigma_{[k-h, k]}^{\dagger}}$ of $\mathcal{O}_{\sigma_{[k-h, k]}}$ and $Q_{\sigma}^{T}$ plays the role of the parametrization.

Besides, according to Corollary $1, y_{k}-\Xi_{s} v_{k}$ fulfills the following successive equalities.

$$
\begin{aligned}
& y_{k}-\Xi_{s} v_{k}=\mathbf{0} \\
& =y_{k}-\left[\begin{array}{lll}
\Xi_{s}^{(1)} & \Xi_{s}^{(2)} & \Xi_{s}^{(3)}
\end{array}\right]\left[\begin{array}{c}
y_{k-h, k-1} \\
u_{k-h, k} \\
\mathbf{1}
\end{array}\right] \\
& =y_{k}-\Xi_{s}^{(1)} y_{k-h, k-1}-\Xi_{s}^{(2)} u_{k-h, k}-\Xi_{s}^{(3)} \\
& =\left[\begin{array}{ll}
-\Xi_{s}^{(1)} & \boldsymbol{1}
\end{array}\right]\left[\begin{array}{c}
y_{k-h, k-1} \\
y_{k}
\end{array}\right] \\
& -\left(\left[C_{\sigma(k)} B_{\sigma(k-1)}^{\sigma(k-h)} \quad D_{\sigma(k)}\right]-\Xi_{s}^{(1)} T_{\sigma_{[k-h, k-1]}}\right) u_{k-h, k} \\
& -\left(\left[C_{\sigma(k)} E_{\sigma(k-1)}^{\sigma(k-h)}\right]-\Xi_{s}^{(1)} T_{\sigma_{[k-h, k-1]}}^{\prime}\right) \\
& =\left[\begin{array}{ll}
-\Xi_{s}^{(1)} & \boldsymbol{1}
\end{array}\right] y_{k-h, k}-\left[\begin{array}{ll}
-\Xi_{s}^{(1)} & \boldsymbol{1}
\end{array}\right] \\
& {\left[\begin{array}{c}
T_{\sigma_{[k-h, k-1]}} \\
C_{\sigma(k)} B_{\sigma(k-1)}^{\sigma(k-h)} D_{\sigma(k)}
\end{array}\right] u_{k-h, k}} \\
& -\left[\begin{array}{ll}
-\Xi_{s}^{(1)} & \boldsymbol{1}
\end{array}\right]\left[\begin{array}{c}
T_{\sigma_{[k-h, k-1]}}^{\prime} \\
C_{\sigma(k)} E_{\sigma(k-1)}^{\sigma(k-h)}
\end{array}\right] \\
& =\left[\begin{array}{ll}
-\Xi_{s}^{(1)} & \boldsymbol{1}
\end{array}\right]\left(y_{k-h, k}-T_{\sigma_{[k-h, k]}} u_{k-h, k}-T_{\sigma_{[k-h, k]}}^{\prime}\right)
\end{aligned}
$$

which completes the proof.

\section{COMPARATIVE STUDY}

Here, we compare the different aforementioned modes detections.

\section{A. Comparison of the structure of the modes detectors}

The aforementioned detectors can be written in a unified way and we have the following proposition.

Proposition 4. The active sequence $\sigma^{*}$ in the interval of time $[k-h, k]$ is a sequence $\sigma_{s} \in \mathcal{J}^{h+1}(s \in \mathcal{S})$ such that the corresponding residual fulfills

$$
r_{h, \sigma_{s}}=\mathbf{0}
$$

with

$$
r_{h, \sigma_{s}}=\Lambda_{\sigma_{s}}\left(y_{k-h, k}-T_{\sigma_{s}} u_{k-h, k}-T_{\sigma_{s}}^{\prime}\right)
$$

where

- $\Lambda_{\sigma_{s}}=\left(\mathcal{O}_{\sigma_{s}} \mathcal{O}_{\sigma_{s}}^{\dagger}-\mathbf{1}\right)$ for the detector 1

- $\Lambda_{\sigma_{s}}=\Omega_{\sigma_{s}}$ for the detector 2

- $\Lambda_{\sigma_{s}}=\left[\begin{array}{ll}H_{s} & 1\end{array}\right]$ for the detector 3

with $\mathcal{O}_{\sigma_{s}}$ defined by (3), $\Omega_{\sigma_{s}}$ the solution of (10) and $H_{s}$ defined by (18).

Proof. See respectively (8), (11) and (17).

Despite the similarities of the structures, some important distinctions must be highlighted. This is the purpose of the next subsection. 


\section{B. Comparison of the size of the horizon}

As it turns out, solving (20) may lead to several solutions $\sigma_{s} \in$ $\mathcal{J}^{h+1}(s \in \mathcal{S})$ and $\sigma^{*}$ correspond to one specific solution. The uniqueness of the solution depends on $\Lambda_{\sigma_{s}}$ which in turn, depends on $h$. For example, let have $\Lambda_{\sigma_{s}}=\mathbf{0}$. In such a case, (20) is always fulfilled and then, any sequence $\sigma_{s}$ causes the residual $r_{h, \sigma_{s}}$ to vanish. Hence, it is clear that $\Lambda_{\sigma_{s}}=\mathbf{0}$ is an admissible solution but is obviously not satisfactory. This being the case, in the following, it is given the minimal size of the horizon to guarantee, at least, the existence of a non zero matrix $\Lambda_{\sigma_{s}}$. Let us note that for the detector 3 , the non nullity is guaranteed by construction. Deriving conditions that guarantee the uniqueness of the solution delivered by the detectors will be the purpose of Section III.

1) Detector 1: The theorem stated in [34] will be useful in the sequel and is recalled below.

Theorem 2. [34] For any matrix $X$, it holds that

$$
\operatorname{ker}(X)=\mathcal{R}\left(\boldsymbol{1}-X^{\dagger} X\right)
$$

Proposition 5. For the detector 1 , the lower bound of the detection horizon is the smallest integer $h$ which fulfills

$$
h>\operatorname{rank}\left(\mathcal{O}_{\sigma_{s}}\right)-1
$$

Proof. For the detector $1, \Lambda_{\sigma_{s}}=\left(\mathcal{O}_{\sigma_{s}} \mathcal{O}_{\sigma_{s}}^{\dagger}-1\right)$. Equation (23) implies that $\operatorname{ker}\left(\mathcal{O}_{\sigma_{s}}\right)$ is not zero. According to Theorem 2, this is equivalent to $\mathcal{R}\left(\boldsymbol{1}-\mathcal{O}_{\sigma_{s}}^{\dagger} \mathcal{O}_{\sigma_{s}}\right)$ different from zero or $\mathcal{R}\left(\mathcal{O}_{\sigma_{s}} \mathcal{O}_{\sigma_{s}}^{\dagger}-\mathbf{1}\right)$ different from zero since by definition of the pseudo-inverse, $\mathcal{O}_{\sigma_{s}}^{\dagger} \mathcal{O}_{\sigma_{s}}$ is symmetric. Finally, this implies that $\left(\mathcal{O}_{\sigma_{s}} \mathcal{O}_{\sigma_{s}}^{\dagger}-1\right)$ and so $\Lambda_{\sigma_{s}}$ is different from zero.

\section{2) Detector 2:}

Proposition 6. For the detector 2, the lower bound of the detection horizon is the smallest integer $h$ which fulfills

$$
h>n-1
$$

Proof. For the detector 2, $\Lambda_{\sigma_{s}} \in \operatorname{ker}^{T}\left(\mathcal{O}_{\sigma_{s}}^{T}\right)$ and it is assumed that the PathWise Observability is satisfied. Since the PathWise Observability is satisfied, $\operatorname{rank}\left(\mathcal{O}_{\sigma_{s}}\right)=\operatorname{rank}\left(\mathcal{O}_{\sigma_{s}}^{T}\right)=n$ which implies that $\operatorname{ker}\left(\mathcal{O}_{\sigma_{s}}^{T}\right)$ is not zero. Hence, it is possible to get $\Lambda_{\sigma_{s}} \in \operatorname{ker}^{T}\left(\mathcal{O}_{\sigma_{s}}^{T}\right)$ different from zero.

3) Detector 3: Unlike the detectors 1 and 2, the existence of $\Lambda_{\sigma_{s}}$ is not always guaranteed since (13) must be fulfilled. On the other hand, if (13) is satisfied, $\Lambda_{\sigma_{s}}$ is not zero by construction. The purpose of this section is to rewrite (13) in terms of the minimal requirement that $h$ must verify to ensure the existence of $\Lambda_{\sigma_{s}}$. First, the following standard lemma is recalled.

Lemma 2. For any two matrices $W$ and $Z$, the equation $W X=Z$, with unknown matrix $X$, admits a solution if and only if $\operatorname{rank}\left(\left[\begin{array}{ll}W & Z\end{array}\right]\right)=\operatorname{rank}(W)\left(\left[\begin{array}{ll}W & Z\end{array}\right]\right.$ stands for the horizontal concatenation of the matrices $W$ and $Z$ ).

The following proposition holds.

Proposition 7. For the detector 3, the lower bound of the detection horizon is the smallest integer $h$ which fulfills

$$
\operatorname{rank}\left(\mathcal{O}_{\sigma_{[k-h, k]}}\right)=\operatorname{rank}\left(\mathcal{O}_{\sigma_{[k-h, k-1]}}\right)
$$

Proof. Consider Lemma 2 with $X=\Xi_{s}^{(1) T}, W=\mathcal{O}_{\sigma_{[k-h, k-1]}^{T}}^{T}$ and $Z=\left(C_{\sigma(k)} A_{\sigma(k-1)}^{\sigma(k-h)}\right)^{T}$. Checking whether (13) has a solution is equivalent to check the rank condition

$\operatorname{rank}\left(\left[\mathcal{O}_{\sigma_{[k-h, k-1]}{ }^{T}}\left(C_{\sigma(k)} A_{\sigma(k-1)}^{\sigma(k-h)}\right)^{T}\right]\right)=\operatorname{rank}\left(\mathcal{O}_{\sigma_{[k-h, k-1]}{ }^{T}}\right.$ which is equivalent to

$$
\operatorname{rank}\left(\left[\begin{array}{c}
\mathcal{O}_{\sigma_{[k-h, k-1]}} \\
C_{\sigma(k)} A_{\sigma(k-1)}^{\sigma(k-h)}
\end{array}\right]\right)=\operatorname{rank}\left(\mathcal{O}_{\sigma_{[k-h, k-1]}}\right)
$$

which, finally, is equivalent to (25). The lower bound of $h$ verifying (25) guarantees that $\Lambda_{\sigma_{s}}$ exists and clearly, if so, $\left[\begin{array}{ll}H_{s} & 1\end{array}\right]$ is different from the zero matrix for every $s \in \mathcal{S}$ by construction.

In other words, the lower bound of the detection horizon must be chosen such that the rank of the observability matrices is kept unchanged considering two horizons of successive length $h$ and $h+1$.

Remark 1. The lower bound $h$ given in [24] for the detector 2 and corresponding to the PathWise Observability, that is to $\operatorname{rank}\left(\mathcal{O}_{\sigma_{[k-h, k]}}\right)=n$, is restrictive and is a particular case of (25) since (25) admits $h<n$ as a solution.

\section{Comparison of the detector 2 and the detector 1}

Proposition 8. For a same size $h$ of the horizon compatible with (23) and (24), the detector 2 is equivalent to the detector 1.

Proof. Taking into account that $\Lambda_{\sigma_{s}}=\left(\mathcal{O}_{\sigma_{s}} \mathcal{O}_{\sigma_{s}}^{\dagger}-1\right)$ for the detector 1 and $\Lambda_{\sigma_{s}}=\Omega_{\sigma_{s}}$ for the detector 2, it is a straightforward consequence of Theorem 2.

\section{Comparison of the detector 3 and the detector 2}

Proposition 9. For a same size $h$ of the horizon compatible with (24) and (25), the detector 3 is a particular case of the detector 2.

Proof. According to Proposition 4, $\Lambda_{\sigma_{s}}=\Omega_{\sigma_{s}}$ for the detector 2 and $\Lambda_{\sigma_{s}}=\left[\begin{array}{ll}H_{s} & 1\end{array}\right]$ for the detector 3. It must be shown that any solution $\Lambda_{\sigma_{s}}=\Omega_{\sigma_{s}}$ of (12) is also a solution of (16) but that the converse is not true. To this end, it must be shown, first, that

$$
\left[\begin{array}{ll}
H_{s} & \boldsymbol{1}
\end{array}\right]^{T} \in \operatorname{ker}\left(\mathcal{O}_{\sigma_{s}}^{T}\right)
$$

The following equalities hold.

$$
\begin{aligned}
\mathcal{O}_{\sigma_{[k-h, k]}^{T}}^{T}\left[\begin{array}{ll}
H_{s} & \boldsymbol{1}
\end{array}\right]^{T} \\
=\left[\begin{array}{c}
\mathcal{O}_{\sigma_{[k-h, k-1]}} \\
C_{\sigma(k)} A_{\sigma(k-1)}^{\sigma(k-h)}
\end{array}\right]^{T}\left[\begin{array}{ll}
-\Xi_{s}^{(1)} & \boldsymbol{1}
\end{array}\right]^{T} \\
=\left[\begin{array}{ll}
\mathcal{O}_{\sigma_{[k-h, k-1]}^{T}}^{T} & \left(C_{\sigma(k)} A_{\sigma(k-1)}^{\sigma(k-h)}\right)^{T}
\end{array}\right]\left[\begin{array}{c}
-\Xi_{s}^{(1) T} \\
\boldsymbol{1}
\end{array}\right] \\
=-\mathcal{O}_{\sigma_{[k-h, k-1]}^{T}}^{T} \Xi_{s}^{(1)^{T}}+\left(C_{\sigma(k)} A_{\sigma(k-1)}^{\sigma(k-h)}\right)^{T} \\
=-\left(\Xi_{s}^{(1)} \mathcal{O}_{\sigma[k-h, k-1]}\right)^{T}+\left(C_{\sigma(k)} A_{\sigma(k-1)}^{\sigma(k-h)}\right)^{T} \\
=-\left(C_{\sigma(k)} A_{\sigma(k-1)}^{\sigma(k-h)}\right)^{T}+\left(C_{\sigma(k)} A_{\sigma(k-1)}^{\sigma(k-h)}\right)^{T} \\
=\mathbf{0}
\end{aligned}
$$

meaning that $\left[\begin{array}{ll}H_{s} & \boldsymbol{1}\end{array}\right]^{T} \in \operatorname{ker}\left(\mathcal{O}_{\sigma_{[k-h, k]}^{T}}^{T}\right)$.

Besides, by definition of $\Omega_{\sigma_{s}}$ as the left null space of $\mathcal{O}_{\sigma_{s}}$, we have that

$$
N_{\text {rows }}\left(\Omega_{\sigma_{s}}\right)=\left(m(h+1)-\operatorname{rank}\left(\mathcal{O}_{\sigma_{s}}\right)\right)
$$

and

$$
N_{\text {rows }}\left(\left[\begin{array}{ll}
H_{s} & \mathbf{1}
\end{array}\right]\right)=m
$$

where $N_{\text {rows }}(X)$ denotes the number of rows of the matrix $X$. As a result, considering the special case when $\operatorname{rank}\left(\mathcal{O}_{\sigma_{s}}\right)<m h$, it holds that

$$
N_{\text {rows }}\left(\Omega_{\sigma_{s}}\right)>N_{\text {rows }}\left(\left[\begin{array}{ll}
H_{s} & \mathbf{1}
\end{array}\right]\right)
$$


Therefore, since $\Omega_{\sigma_{s}}^{T}$ is full column rank by definition of a left null space, in this case, a solution of the detector 2 cannot be a solution of the detector 3. That completes the proof.

Remark 2. In the particular case when $\operatorname{rank}\left(\mathcal{O}_{\sigma_{s}}\right)=m$, the detector 3 is equivalent to the detector 2. Indeed, in such a case, $N_{\text {rows }}\left(\Omega_{\sigma_{s}}\right)=N_{\text {rows }}\left(\left[\begin{array}{ll}H_{s} & \boldsymbol{1}\end{array}\right]\right)$.

As $\Omega_{\sigma_{s}}$ only involves independent rows by definition of a left null space, so does $\left[\begin{array}{ll}H_{s} & 1\end{array}\right]$ since

$$
\operatorname{rank}\left(\left[\begin{array}{ll}
H_{s} & \boldsymbol{1}
\end{array}\right]\right)=\operatorname{rank}(\boldsymbol{1})=N_{\text {rows }}\left(\left[\begin{array}{ll}
H_{s} & \boldsymbol{1}
\end{array}\right]\right)
$$

Let us note that the condition $\operatorname{rank}\left(\mathcal{O}_{\sigma_{s}}\right)>m h$ cannot be verified since $h$ obeys condition (25), $\operatorname{rank}\left(\mathcal{O}_{\sigma_{s}}\right) \leq n$ and $m>0$.

\section{E. Conclusion on detectors}

In the light of the above comparative study, we can define a general detector which encompasses all of aforementioned detectors and the following proposition applies.

Proposition 10. Let $h$ be the smallest integer fulfilling

$$
\operatorname{rank}\left(\mathcal{O}_{\sigma_{[k-h, k]}}\right)=\operatorname{rank}\left(\mathcal{O}_{\sigma_{[k-h, k-1]}}\right)
$$

The active sequence $\sigma^{*}$ in the interval of time $[k-h, k]$ is a sequence $\sigma_{s} \in \mathcal{J}^{h+1}(s \in \mathcal{S})$ such that the corresponding residual fulfills

$$
r_{h, \sigma_{s}}=\mathbf{0}
$$

with

$$
r_{h, \sigma_{s}}=\Omega_{\sigma_{s}}\left(y_{k-h, k}-T_{\sigma_{s}} u_{k-h, k}-T_{\sigma_{s}}^{\prime}\right),
$$

where $\Omega_{\sigma_{s}}$ is the solution of

$$
\Omega_{\sigma_{s}} \mathcal{O}_{\sigma_{s}}=\mathbf{0}
$$

Proof. The kernel $\Omega_{\sigma_{s}}$ of $\mathcal{O}_{\sigma_{s}}$ encompasses of the solutions $\Lambda_{\sigma_{s}}$ summed up in Proposition 4 as proved in Proof II-C and Proof II-D. The horizon length $h$ satisfying (27) encompasses all the other conditions (23) and (24) as pointed out in Remark 1.

As a straightforward consequence, the following corollary applies to estimate $\sigma^{*}(k)$.

Corollary 2. The active mode $\sigma^{*}(k)$ is the last element of one of the sequences $\sigma_{s}$ which are solutions of (28).

In the sequel, we are interested in the important question which arises regarding the detector: its ability of delivering a unique solution $\sigma^{*}(k)$. Since, the solutions of the modes detector, owing to its structure inherent to the discrete-time case, are sequences of modes, it is sufficient to check whether the modes detector is able to discriminate the active sequence $\sigma^{*}$ from any other sequences $\sigma_{s} \in \mathcal{J}^{h+1}(s \in \mathcal{S})$. Such a notion is related to the so-called discernibility addressed in the section below. Then, we will show in Section IV that although the discernibility is not verified, we can resort to alternatives to uniquely estimate $\sigma^{*}(k)$.

\section{DISCERNIBILITY}

\section{A. Conditions for discernibility}

Discernibility has been thoroughly discussed in [23] and [24]. Formally, discernibility of modes sequences obeys the following definition which is restated here to be in accordance with the notation of the present paper.

The two following definitions are general. They are only based on residuals without considering a specific structure of the modes detectors.
Definition 2. [24] Let us consider two modes sequences $\sigma_{s_{i}}$ and $\sigma_{s_{j}}$ delivered by (1), with $\left(s_{i}, s_{j}\right) \in \mathcal{S}^{2}$. Assume that $\sigma_{s_{i}}$ is the active sequence. The sequence $\sigma_{s_{i}}$ is discernable from $\sigma_{s_{j}}$ on an observation window of length $h+1$, if the corresponding residuals $r_{h, \sigma_{s_{i}}}$ and $r_{h, \sigma_{s_{j}}}$ are not simultaneously zero.

We can introduce the notion of discernibility for the system (1).

Definition 3. The system (1) is discernable if, for every pairs of sequences $\sigma_{s_{i}} \in \mathcal{J}^{h+1}, \sigma_{s_{j}} \in \mathcal{J}^{h+1}$ with $\left(s_{i}, s_{j}\right) \in \mathcal{S}^{2}, \sigma_{s_{i}}$ is discernable from $\sigma_{s_{j}}$.

In the sequel, conditions are particularized to the general detector defined as in Proposition 10.

A necessary and sufficient condition for discernibility is proved in [24] and is recalled below.

Theorem 3. [24] Let us consider two modes sequences $\sigma_{s_{i}}$ and $\sigma_{s_{j}}$ delivered by (1), with $\left(s_{i}, s_{j}\right) \in \mathcal{S}^{2}$. Assume that $\sigma_{s_{i}}$ is the active sequence. For a given input sequence $u_{k-h, k}, \sigma_{s_{i}}$ is discernable from $\sigma_{s_{j}}$ on an observation window of length $h+1$, for almost all initial conditions $x_{k-h}$, iff at least one of the two following conditions is satisfied

$$
\begin{gathered}
\Omega_{\sigma_{s_{j}}} \mathcal{O}_{\sigma_{s_{i}}} \neq \mathbf{0} \\
\Omega_{\sigma_{s_{j}}}\left(\left(T_{\sigma_{s_{i}}}-T_{\sigma_{s_{j}}}\right) u_{k-h, k}+\left(T_{\sigma_{s_{i}}}^{\prime}-T_{\sigma_{s_{j}}}^{\prime}\right)\right) \neq \mathbf{0}
\end{gathered}
$$

The following remarks can be made.

Remark 3. The question of the existence of the control sequences $u_{k-h, k}$ which satisfy (30) is not trivial. It has been addressed in [30], [31] where the notions of "discerning control sequences" and "active observability" have been introduced.

Remark 4. Condition (29) is a necessary and sufficient conditions for discernibility when considering uncontrolled switched linear systems or uncontrolled switched affine systems with a constant affine part and a constant output matrix. Indeed, for uncontrolled systems, the input matrix and the direct transfer matrix are zero, inducing $T_{\sigma_{s}}=\mathbf{0}$. Besides, for switched linear systems, $T_{\sigma_{s}}^{\prime}$ is zero and for switched affine systems with a constant affine part and a constant output matrix, the difference $T_{\sigma_{s_{i}}}^{\prime}-T_{\sigma_{s_{j}}}^{\prime}$ is zero.

As a straightforward consequence, the following corollary applies to estimate $\sigma^{*}(k)$.

Corollary 3. Assume that the system (1) is discernable. The active mode $\sigma^{*}(k)$ is the last element of the sequence $\sigma_{s}$ which is the unique solution of (28).

Conditions (29) and (30) depend on the structure of the detector since they involve the matrices $\Omega_{\sigma_{s_{j}}}$. An equivalent condition of (29) that holds regardless of the structure of the detector and does not require the computation of $\Omega_{\sigma_{s_{j}}}$ is proposed below.

Proposition 11. Condition (29) is equivalent to state that

$$
\operatorname{rank}\left(\left[\begin{array}{ll}
\mathcal{O}_{\sigma_{s_{i}}} & \mathcal{O}_{\sigma_{s_{j}}}
\end{array}\right]\right)>\operatorname{rank}\left(\mathcal{O}_{\sigma_{s_{j}}}\right)
$$

where $\left[\begin{array}{ll}\mathcal{O}_{\sigma_{s_{i}}} & \mathcal{O}_{\sigma_{s_{j}}}\end{array}\right]$ denotes the horizontal concatenation of $\mathcal{O}_{\sigma_{s_{i}}}$ and $\mathcal{O}_{\sigma_{s_{j}}}$.

Proof. A reductio ad absurdum reasoning is performed. If (31) is not satisfied, it is equivalent to

$$
\operatorname{rank}\left(\left[\begin{array}{ll}
\mathcal{O}_{\sigma_{s_{i}}} & \mathcal{O}_{\sigma_{s_{j}}}
\end{array}\right]\right)=\operatorname{rank}\left(\mathcal{O}_{\sigma_{s_{j}}}\right)
$$

since it is clear that $\operatorname{rank}\left(\left[\begin{array}{ll}\mathcal{O}_{\sigma_{s_{i}}} & \mathcal{O}_{\sigma_{s_{j}}}\end{array}\right]\right)<\operatorname{rank}\left(\mathcal{O}_{\sigma_{s_{j}}}\right)$ is not admissible. This means that $\mathcal{O}_{\sigma_{s_{i}}} \in \mathcal{R}\left(\mathcal{O}_{\sigma_{s_{j}}}\right)$. From (7) of Lemma 1, 
we get that

$$
\mathcal{O}_{\sigma_{s_{i}}} \in \mathcal{R}\left(\mathcal{O}_{\sigma_{s_{j}}}\right) \Leftrightarrow\left(\mathcal{O}_{\sigma_{s_{j}}} \mathcal{O}_{\sigma_{s_{j}}}^{\dagger}-\mathbf{I}\right) \mathcal{O}_{\sigma_{s_{i}}}=\mathbf{0}
$$

The following equivalence holds

$$
\begin{gathered}
\left(\mathcal{O}_{\sigma_{s_{j}}} \mathcal{O}_{\sigma_{s_{j}}}^{\dagger}-\boldsymbol{1}\right) \mathcal{O}_{\sigma_{s_{i}}}=\mathbf{0} \\
\Leftrightarrow \\
\left(1-\mathcal{O}_{\sigma_{s_{j}}} \mathcal{O}_{\sigma_{s_{j}}}^{\dagger}\right) \mathcal{O}_{\sigma_{s_{i}}}=\mathbf{0} \\
\Leftrightarrow \\
\mathcal{O}_{\sigma_{s_{i}}}^{T}\left(\boldsymbol{1}-\mathcal{O}_{\sigma_{s_{j}}}^{\dagger} \mathcal{O}_{\sigma_{s_{j}}}^{T}\right)=\mathbf{0}
\end{gathered}
$$

According to Theorem 2, the last equality is equivalent to $\mathcal{O}_{\sigma_{s_{i}}}^{T} \operatorname{ker}\left(\mathcal{O}_{\sigma_{s_{j}}}^{T}\right)=\mathbf{0}$. Therefore, since by definition $\Omega_{\sigma_{s_{j}}}^{T} \in$ $\operatorname{ker}\left(\mathcal{O}_{\sigma_{s_{j}}}^{T}\right)$, this implies that

$$
\Omega_{\sigma_{s_{j}}} \mathcal{O}_{\sigma_{s_{i}}}=\mathbf{0}
$$

That completes the proof.

Let us note that condition (31) corresponds to the definition of discernibility proposed in [29]. Actually, such a definition is a sufficient condition of discernibility since (30) must be considered as well. However, it is a necessary and sufficient condition for systems verifying the conditions highlighted in Remark 4. The consideration of Theorem 3 and of Proposition 11 yields the corollary given below.

Corollary 4. For two sequences $\sigma_{s_{i}} \in \mathcal{J}^{h+1}\left(s_{i} \in \mathcal{S}\right)$ and $\sigma_{s_{j}} \in$ $\mathcal{J}^{h+1}\left(s_{j} \in \mathcal{S}\right)$, the following relations hold

$$
\begin{gathered}
\operatorname{rank}\left(\left[\begin{array}{cc}
\mathcal{O}_{\sigma_{s_{i}}} & \mathcal{O}_{\sigma_{s_{j}}}
\end{array}\right]\right)>\operatorname{rank}\left(\mathcal{O}_{\sigma_{s_{j}}}\right) \\
\Leftrightarrow \\
\Omega_{\sigma_{s_{j}}} \mathcal{O}_{\sigma_{s_{i}}} \neq \mathbf{0} \\
\Rightarrow \\
\sigma_{s_{i}} \text { is discernable from } \sigma_{s_{j}}
\end{gathered}
$$

The last inference turns into an equivalence for systems verifying the conditions highlighted in Remark 4.

Proposition 12. Consider two modes sequences $\sigma_{s_{i}}$ and $\sigma_{s_{j}}$ with $\left(s_{i}, s_{j}\right) \in \mathcal{S}^{2}$ and $j \neq i$. The two following statements are not equivalent

A1) $\sigma_{s_{i}}$ is discernable from $\sigma_{s_{j}}$

A2) $\sigma_{s_{j}}$ is discernable from $\sigma_{s_{i}}$

It is said that discernibility is not reversible.

Proof. Let us show that although $\sigma_{s_{i}}$ is not discernable from $\sigma_{s_{j}}$, $\sigma_{s_{j}}$ may be discernable from $\sigma_{s_{i}}$.

Consider the special case when $\Omega_{\sigma_{s_{i}}}=\operatorname{ker}\left(\mathcal{O}_{\sigma_{s_{i}}}\right)$ and $\Omega_{\sigma_{s_{j}}}=$ $\operatorname{ker}\left(\mathcal{O}_{\sigma_{s_{j}}}\right)$. If $\sigma_{s_{i}}$ is not discernable from $\sigma_{s_{j}}$ then

$$
\Omega_{\sigma_{s_{j}}} \mathcal{O}_{\sigma_{s_{i}}}=\mathbf{0}
$$

Since by definition, $\Omega_{\sigma_{s_{i}}} \mathcal{O}_{\sigma_{s_{i}}}=\mathbf{0}$, it holds that

$$
\operatorname{rank}\left(\operatorname{ker}\left(\mathcal{O}_{\sigma_{s_{i}}}^{T}\right)\right)=\operatorname{rank}\left(\Omega_{\sigma_{s_{i}}}^{T}\right) \geq \operatorname{rank}\left(\Omega_{\sigma_{s_{j}}}^{T}\right)
$$

Now, let us consider the special case when $\operatorname{rank}\left(\Omega_{\sigma_{s_{i}}}^{T}\right) \neq$ $\operatorname{rank}\left(\Omega_{\sigma_{s_{j}}}^{T}\right)$. Then, it holds that

$$
\operatorname{rank}\left(\Omega_{\sigma_{s_{i}}}^{T}\right)>\operatorname{rank}\left(\Omega_{\sigma_{s_{j}}}^{T}\right)
$$

Equation (35) implies that $\Omega_{\sigma_{s_{i}}}^{T}$ does not span $\operatorname{ker}\left(\mathcal{O}_{\sigma_{s_{j}}}^{T}\right)$. Therefore, it is clear that

$$
\Omega_{\sigma_{s_{i}}} \mathcal{O}_{\sigma_{s_{j}}} \neq \mathbf{0}
$$

As a result, based on Corollary $4, \sigma_{s_{j}}$ is discernable from $\sigma_{s_{i}}$. That completes the proof.

\section{B. Measure of discernibility}

Three main quantities have been proposed in the literature to assess whether discernibility is "strong" or "weak": the "mode observability", the "degree of discernibility" and the "conflict between two sequences". This allows to give a measure of the "distance" between two sequences that are intended to be discriminated. All of those quantities are related to (31) and so, provide sufficient conditions for controlled systems. On the other hand, they do not depend on the structure of the detector. They are recalled and compared below.

1) degree of discernibility

In [29], the degree of discernibility is defined as the positive integer $d>0$ satisfying

$$
d=\operatorname{rank}\left(\left[\begin{array}{ll}
\mathcal{O}_{\sigma_{s_{i}}} & \mathcal{O}_{\sigma_{s_{j}}}
\end{array}\right]\right)-\operatorname{rank}\left(\mathcal{O}_{\sigma_{s_{j}}}\right)
$$

Condition (31) can be expressed in terms of the degree of discernibility. When (31) is fulfilled, it is said that $\sigma_{s_{i}}$ is $d$-discernable from $\sigma_{s_{j}}$ with degree of discernibility equal to $d$. When $d=0$, it means that $\sigma_{s_{i}}$ is not discernable from $\sigma_{s_{j}}$. It is interesting to stress that when $\sigma_{s_{i}}$ is $d$-discernable from $\sigma_{s_{j}}, \sigma_{s_{j}}$ is likely to be discernable from $\sigma_{s_{i}}$ with a different degree of discernibility. That holds in the special situation when $\operatorname{rank}\left(\mathcal{O}_{\sigma_{s_{i}}}\right) \neq \operatorname{rank}\left(\mathcal{O}_{\sigma_{s_{i}}}\right) \neq 0$. That corroborates the non reversibility of the discernibility as highlighted by Proposition 12 .

Let us notice that a close notion has been introduced in [27], [28] under the name degree of distinguishability at $x$ and is suitable to cope with noisy measurements. The notion combines elements from canonical correlation analysis and subspace projection methods.

2) conflict between two sequences

In [29], the conflict of $\sigma_{s_{i}}$ with $\sigma_{s_{j}}$ is given by the quantity $C\left(\sigma_{s_{i}}, \sigma_{s_{j}}\right)$ defined as

$$
C\left(\sigma_{s_{i}}, \sigma_{s_{j}}\right)=\mathcal{R}\left(\mathcal{O}_{\sigma_{s_{i}}}\right) \cap \mathcal{R}\left(\mathcal{O}_{\sigma_{s_{j}}}\right)
$$

The dimension of the conflict is given by the quantity $\operatorname{dim}\left(C\left(\sigma_{s_{i}}, \sigma_{s_{j}}\right)\right)$ defined as

$$
\begin{gathered}
\operatorname{dim}\left(C\left(\sigma_{s_{i}}, \sigma_{s_{j}}\right)\right)=\operatorname{rank}\left(\mathcal{O}_{\sigma_{s_{i}}}\right)+\operatorname{rank}\left(\mathcal{O}_{\sigma_{s_{j}}}\right) \\
-\operatorname{rank}\left(\left[\begin{array}{ll}
\mathcal{O}_{\sigma_{s_{i}}} & \mathcal{O}_{\sigma_{s_{j}}}
\end{array}\right]\right)
\end{gathered}
$$

It follows that

$$
\begin{gathered}
\operatorname{dim}\left(C\left(\sigma_{s_{i}}, \sigma_{s_{j}}\right)\right)=0 \Leftrightarrow \\
\operatorname{rank}\left(\left[\begin{array}{cc}
\mathcal{O}_{\sigma_{s_{i}}} & \mathcal{O}_{\sigma_{s_{j}}}
\end{array}\right]\right)=\operatorname{rank}\left(\mathcal{O}_{\sigma_{s_{i}}}\right)+\operatorname{rank}\left(\mathcal{O}_{\sigma_{s_{j}}}\right)
\end{gathered}
$$

When $\operatorname{dim}\left(C\left(\sigma_{s_{i}}, \sigma_{s_{j}}\right)\right)=0$, there is no conflict and, provided that $\operatorname{rank}\left(\mathcal{O}_{\sigma_{s_{i}}}\right) \neq 0$ and $\operatorname{rank}\left(\mathcal{O}_{\sigma_{s_{j}}}\right) \neq 0$, by virtue of (31), $\sigma_{s_{i}}$ is discernable from $\sigma_{s_{j}}$ and $\sigma_{s_{j}}$ is discernable from $\sigma_{s_{i}}$. This reversibility highlights that (36) is a sufficient condition for (31) to hold but is not necessary. It is explained by the fact that a sequence $\sigma_{s_{i}}$ can be discernable from another sequence $\sigma_{s_{j}}$ although there is a conflict, that is $\operatorname{dim}\left(C\left(\sigma_{s_{i}}, \sigma_{s_{j}}\right)\right) \neq 0$. Recalling that $\sigma_{s_{i}}$ is discernable from $\sigma_{s_{j}}$ implies that the degree of discernibility $d$ is such that $d>0$ and noticing that $d=\operatorname{rank}\left(\mathcal{O}_{\sigma_{s_{i}}}\right)-\operatorname{dim}\left(C\left(\sigma_{s_{i}}, \sigma_{s_{j}}\right)\right), \sigma_{s_{i}}$ is discernable from $\sigma_{s_{j}}$ whenever $\operatorname{dim}\left(C\left(\sigma_{s_{i}}, \sigma_{s_{j}}\right)\right) \leq \operatorname{rank}\left(\mathcal{O}_{\sigma_{s_{i}}}\right)-1$. Actually, $\operatorname{dim}\left(C\left(\sigma_{s_{i}}, \sigma_{s_{j}}\right)\right)=0$ corresponds to the special case $d=\operatorname{rank}\left(\mathcal{O}_{\sigma_{s_{i}}}\right)$. As a result, the conflict between two sequences gives a more restrictive condition than the degree of discernibility.

3) mode observability

Mode observability is considered under different terminologies in [25] [20] and allows to give a condition on discernibility according the following proposition. 
Proposition 13. [25], [20] Two any sequences $\sigma_{s_{i}}$ and $\sigma_{s_{j}}$ with $\left(s_{i}, s_{j}\right) \in \mathcal{S}^{2}$ are discernable if

$$
\operatorname{rank}\left(\left[\begin{array}{ll}
\mathcal{O}_{\sigma_{s_{i}}} & \mathcal{O}_{\sigma_{s_{j}}}
\end{array}\right]\right)=2 n
$$

The matrix $\left[\begin{array}{ll}\mathcal{O}_{\sigma_{s_{i}}} & \mathcal{O}_{\sigma_{s_{j}}}\end{array}\right]$ is called the joint observability matrix. Condition (37) requires that $\operatorname{rank}\left(\mathcal{O}_{\sigma_{s}}\right)=n$ for all $s \in\left\{s_{i}, s_{j}\right\}$ with $\left(s_{i}, s_{j}\right) \in \mathcal{S}^{2}$, that is, the system must be PathWise Observable. Note that PathWise Observability is not equivalent to mode observability. It is only a necessary condition since it may happen that a system is PathWise Observable and that $\mathcal{R}\left(\mathcal{O}_{\sigma_{s_{i}}}\right) \cap \mathcal{R}\left(\mathcal{O}_{\sigma_{s_{j}}}\right) \neq \emptyset$ leading to $\operatorname{rank}\left(\left[\mathcal{O}_{\sigma_{s_{i}}} \mathcal{O}_{\sigma_{s_{j}}}\right]\right)<2 n$. Mode observability means that the intersection of the observability subspaces of each pair of observability matrix involved in the joint observability matrix has to be empty. As a result, the mode observability gives a more restrictive condition than the conflict between two sequences.

According to Definition 2, if discernibility is not satisfied, this means that several residuals can vanish simultaneously. That may cause the estimation of the sequence $\sigma_{s}$ as in Proposition 10 or even worse the estimation of the active mode $\sigma^{*}(k)$ as in Corollary 3 to be unsuccessful since the uniqueness is not satisfied. Next section is devoted to the solutions that allow to circumvent such a point, the horizon length being kept unchanged, that is $h$ still verifying (27).

\section{THE CASE OF NON DISCERNABLE SYSTEMS}

Let us introduce $\mathcal{R}_{\sigma_{s_{i}}}\left(s_{i} \in \mathcal{S}\right)$ as the set of sequences $\sigma_{s_{j}}\left(s_{j} \in\right.$ $\mathcal{S})$ such that $\sigma_{s_{i}}$ is not discernable from $\sigma_{s_{j}}$. Let us point out that in the special situations highlighted in Remark $4, \mathcal{R}_{\sigma_{s_{i}}}$ can be defined, according to (31), as

$$
\mathcal{R}_{\sigma_{s_{i}}}=\left\{\sigma_{s_{j}}: s_{j} \in \mathcal{S} \text { and } \operatorname{rank}\left(\left[\begin{array}{ll}
\mathcal{O}_{\sigma_{s_{i}}} & \mathcal{O}_{\sigma_{s_{j}}}
\end{array}\right]\right)=\operatorname{rank}\left(\mathcal{O}_{\sigma_{s_{j}}}\right)\right\}
$$

We must define for the sequel $\mathcal{R}_{\sigma^{*}}$ as the set of all sequences having a residual equal to zero

$$
\mathcal{R}_{\sigma^{*}}=\left\{\sigma_{s_{j}}: s_{j} \in \mathcal{S} \text { and } r_{h, \sigma_{s_{j}}}=\mathbf{0}\right\}
$$

\section{A. $\mathcal{R}$-discernibility}

The $\mathcal{R}$-discernibility is proposed in this paper as an extension to the discernibility. Whenever satisfied, it will allow to discriminate an active sequence from all the other ones although several residuals vanish simultaneously. It is defined below.

Definition 4. Let us consider two modes sequences $\sigma_{s_{i}}$ and $\sigma_{s_{j}}$ delivered by (1), with $\left(s_{i}, s_{j}\right) \in \mathcal{S}^{2}$. Assume that $\sigma_{s_{i}}$ is the active sequence. The sequence $\sigma_{s_{i}}$ is $\mathcal{R}$-discernable from $\sigma_{s_{j}}$ on an observation window of length $h+1$, for almost all initial conditions $x_{k-h}$ if

$$
\mathcal{R}_{\sigma_{s_{i}}} \neq \mathcal{R}_{\sigma_{s_{j}}}
$$

Let us note that $\sigma_{s_{i}}$ is always an element of $\mathcal{R}_{\sigma_{s_{i}}}$ and when the discernibility is satisfied, $\sigma_{s_{i}}$ is the unique element of $\mathcal{R}_{\sigma_{s_{i}}}$. As a consequence, for discernable systems, (40) is always satisfied meaning that the discernibility implies the $\mathcal{R}$-discernibility.

We can introduce the notion of $\mathcal{R}$-discernibility for the system (1).

Definition 5. The system (1) is $\mathcal{R}$-discernable if, for every pairs of sequences $\sigma_{s_{i}} \in \mathcal{J}^{h+1}, \sigma_{s_{j}} \in \mathcal{J}^{h+1}$ with $\left(s_{i}, s_{j}\right) \in \mathcal{S}^{2}$, condition (40) is satisfied.

Proposition 14. Assume that (1) is $\mathcal{R}$-discernable. The active sequence $\sigma^{*}$ in the interval of time $[k-h, k]$ is the unique sequence $\sigma_{s} \in \mathcal{J}^{h+1}(s \in \mathcal{S})$ such that

$$
\mathcal{R}_{\sigma^{*}}=\mathcal{R}_{\sigma_{s}}
$$

\section{Proof. It is a straightforward consequence of Definition 4.}

In other words, the discrimination, and so the estimation, of an active sequence from all the other ones can be achieved if the set $\mathcal{R}_{\sigma^{*}}$ including the active sequence differs from all the other sets $\mathcal{R}_{\sigma_{s_{j}}}$ with $\sigma_{s_{j}} \in \mathcal{J}^{h+1}\left(s_{j} \in \mathcal{S}\right)$. As a straightforward consequence, the following corollary applies to estimate $\sigma^{*}(k)$.

Corollary 5. Assume that (1) is $\mathcal{R}$-discernable. The active mode $\sigma^{*}(k)$ is the last element of $\sigma_{s}$ which is solution of (41).

In the case when the discernibility and the $\mathcal{R}$-discernibility are not satisfied, the whole active sequence of length $h+1$ cannot be discriminated from all the other ones. Hence, the same point holds for the estimation of the active mode. This being the case, an alternative has been proposed through the concept of $(\eta, \omega)$-discernibility [20]. It reflects the ability of discriminating, and so estimating, a part of the active sequence. It is the purpose of next subsection. We will also show how to estimate the active mode from the active subsequence.

\section{B. $(\eta, \omega)$-Discernibility}

Denote with $\sigma_{s[k-h+\eta, k-\omega]}$ the subsequence $\sigma(k-h+$ $\eta), \ldots, \sigma(k-\omega)$ of $\sigma_{s}(s \in \mathcal{S})$. The quantities $\eta$ and $\omega$ are integers verifying $\eta \in\{0, \ldots, h\}, \omega \in\{0, \ldots, h\}$ and $\eta+\omega \leq h$. The property of $(\eta, \omega)$-discernibility [20] reflects the ability of discriminating the active subsequence $\sigma_{[k-h+\eta, k-\omega]}^{*}$ from all the other ones. Clearly, the $(\eta, \omega)$-discernibility coincides with the discernibility when $\eta=0$ and $\omega=0$. In the sequel, we will consider either $\eta>0$ or $\omega>0$. The $(\eta, \omega)$-discernibility obeys the following definition.

Definition 6. [20] $\sigma_{s_{i}}$ is $(\eta, \omega)$-discernable from $\sigma_{s_{j}}$ with $\left(s_{i}, s_{j}\right) \in$ $\mathcal{S}^{2}$ if $\sigma_{s_{i}[k-h+\eta, k-\omega]} \neq \sigma_{s_{j}[k-h+\eta, k-\omega]}$ and $\sigma_{s_{i}}$ is discernable from $\sigma_{s_{j}}$

We can introduce the notion of $(\eta, \omega)$-discernibility for the system (1).

Definition 7. The system (1) is ( $\eta, \omega)$-discernable, if, for every pairs of sequences $\sigma_{s_{i}} \in \mathcal{J}^{h+1}, \sigma_{s_{j}} \in \mathcal{J}^{h+1}$ with $\left(s_{i}, s_{j}\right) \in \mathcal{S}^{2}, \sigma_{s_{i}}$ is $(\eta, \omega)$-discernible from $\sigma_{s_{j}}$.

Based on Theorem 3 of discernibility, we can derive conditions for checking the $(\eta, \omega)$-discernibility. They are given in the following proposition.

Proposition 15. Let us consider two modes sequences $\sigma_{s_{i}}$ and $\sigma_{s_{j}}$ delivered by (1), with $\left(s_{i}, s_{j}\right) \in \mathcal{S}^{2}$. Assume that $\sigma_{s_{i}}$ is the active sequence. For a given input sequence $u_{k-h, k}, \sigma_{s_{i}}$ is $(\eta, \omega)-$ discernable from $\sigma_{s_{j}}$ on an observation window of length $h+1$, for almost all initial conditions $x_{k-h}$, iff at least one of the two following conditions is satisfied

$$
\begin{gathered}
\Omega_{\sigma_{s_{j}}} \mathcal{O}_{\sigma_{s_{i}}} \neq \mathbf{0} \\
\Omega_{\sigma_{s_{j}}}\left(\left(T_{\sigma_{s_{i}}}-T_{\sigma_{s_{j}}}\right) u_{k-h, k}+\left(T_{\sigma_{s_{i}}}^{\prime}-T_{\sigma_{s_{j}}}^{\prime}\right)\right) \neq \mathbf{0} \\
\text { with } \quad \sigma_{s_{j}[k-h+\eta, k-\omega]} \neq \sigma_{s_{i}[k-h+\eta, k-\omega]}
\end{gathered}
$$

Proof. The proof is a straightforward consequence of the consideration of both Definition 6 of the $(\eta, \omega)$-discernibility and (29)-(30) of Theorem 3.

The estimation of the active subsequence $\sigma^{*}{ }_{[k-h+\eta, k-\omega]}$ can be performed according to the following proposition.

Proposition 16. Assume that (1) is $(\eta, \omega)$-discernable. The active subsequence $\sigma^{*}{ }_{[k-h+\eta, k-\omega]}$ in the interval of time $[k-h, k]$ is the unique subsequence $\sigma_{s[k-h+\eta, k-\omega]}$ of the sequences $\sigma_{s} \in \mathcal{J}^{h+1}$ such that

$$
\sigma_{s} \in \mathcal{R}_{\sigma^{*}}
$$


Proof. The set $\mathcal{R}_{\sigma^{*}}$ contains all the sequences $\sigma_{s}$ having the residual $r_{h, \sigma_{s}}$ equal to zero. Therefore, the set $\mathcal{R}_{\sigma^{*}}$ contains all the sequences $\sigma_{s_{j}}$ such that $\sigma^{*}$ is not discernible from $\sigma_{s_{j}}$. However, since the $(\eta, \omega)$-discernibility is verified, it means that all those sequences share a common subsequence $\sigma_{s[k-h+\eta, k-\omega]}$. Consequently, $\sigma_{s[k-h+\eta, k-\omega]}$ is unique and corresponds to $\sigma^{*}[k-h+\eta, k-\omega]$.

As a straightforward consequence, the following corollary applies to estimate $\sigma^{*}(k)$.

Corollary 6. Assume that (1) is $(\eta, \omega)$-discernable. The active mode $\sigma^{*}(k)$ is the $h+1-\omega$ element of the sequence $\sigma_{s}$ which is solution of (44) at time $k+\omega$.

Let us note that there exists a delay equal to $\omega$ between the time instants of the estimation and of the active mode. This is inherent to the $(\eta, \omega)$-discernibility. It is interesting to stress that such a notion is an extension of the so-called Forward Discernibility introduced in [29]. Indeed, the Forward Discernibility reflects the ability to determine a prescribed number $\lambda^{\prime}$ of modes $\sigma(k-h) \ldots \sigma\left(k-\lambda^{\prime}\right)$ in a sequence of length $h+1$. Therefore, the Forward Discernibility is equivalent to the $\left(\eta=0, \omega=\lambda^{\prime}\right)$-Discernibility.

\section{C. $\mathcal{R}(\eta, \omega)$-discernibility}

When the $(\eta, \omega)$-discernibility is not satisfied, the active subsequence $\sigma_{[k-h+\eta, k-\omega]}^{*}$ may however be estimated. The estimation can be achieved under a condition which is an extension of the $(\eta, \omega)$ discernibility. We will call it the $\mathcal{R}(\eta, \omega)$-discernibility.

Let us recall that $\mathcal{R}_{\sigma_{s_{i}}}\left(s_{i} \in \mathcal{S}\right)$ is the set of sequences $\sigma_{s_{j}}\left(s_{j} \in \mathcal{S}\right)$, such that $\sigma_{s_{i}}$ is not discernable from $\sigma_{s_{j}}$.

Definition 8. Let us consider two modes sequences $\sigma_{s_{i}}$ and $\sigma_{s_{j}}$ delivered by (1), with $\left(s_{i}, s_{j}\right) \in \mathcal{S}^{2}$. Assume that $\sigma_{s_{i}}$ is the active sequence. Then, a sequence $\sigma_{s_{i}}$ is $\mathcal{R}(\eta, \omega)$-discernable from $\sigma_{s_{j}}$ on an observation window of length $h+1$, for almost all initial conditions $x_{k-h}$ if

$$
\mathcal{R}_{\sigma_{s_{i}}} \neq \mathcal{R}_{\sigma_{s_{j}}} \text { with } \sigma_{s_{i}[k-h+\eta, k-\omega]} \neq \sigma_{s_{j}[k-h+\eta, k-\omega]}
$$

Let $\mathcal{R}_{\sigma^{*}}^{\prime}$ be the set defined as

$$
\mathcal{R}_{\sigma^{*}}^{\prime}=\left\{\sigma_{s_{j}}: s_{j} \in \mathcal{S} \text { and } \mathcal{R}_{\sigma^{*}}=\mathcal{R}_{\sigma_{s_{j}}}\right\}
$$

The following proposition allows to perform the estimation of $\sigma^{*}[k-h+\eta, k-\omega]$.

Proposition 17. Assume that (1) is $\mathcal{R}(\eta, \omega)$-discernable. The active subsequence $\sigma^{*}{ }_{[k-h+\eta, k-\omega]}$ in the interval of time $[k-h, k]$ is the unique subsequence $\sigma_{s[k-h+\eta, k-\omega]}(s \in \mathcal{S})$ of a sequence $\sigma_{s}$ such that

$$
\sigma_{s} \in \mathcal{R}_{\sigma^{*}}^{\prime}
$$

Proof. The set $\mathcal{R}_{\sigma^{*}}^{\prime}$ contains all the sequences $\sigma_{s_{j}}$ such that $\mathcal{R}_{\sigma^{*}}=\mathcal{R}_{\sigma_{s_{j}}}$ and therefore all the sequences $\sigma_{s_{j}}$ such that $\sigma^{*}$ is not $\mathcal{R}$-discernible from $\sigma_{s_{j}}$. However, since the $\mathcal{R}(\eta, \omega)$ discernibility is verified, all these sequences share a common part $\sigma_{s[k-h+\eta, k-\omega]}$. Consequently, $\sigma_{s[k-h+\eta, k-\omega]}$ is unique and corresponds to $\sigma^{*}{ }_{[k-h+\eta, k-\omega]}$.

As a straightforward consequence, the following corollary applies to estimate $\sigma^{*}(k)$.

Corollary 7. Assume that (1) is $\mathcal{R}(\eta, \omega)$-discernable. The active mode $\sigma^{*}(k)$ is the $h+1-\omega$ element of the sequence $\sigma_{s}$ which is solution of (47) at time $k+\omega$.

Likewise for the $(\eta, \omega)$-discernibility, there is an inherent delay equal to $\omega$.

\section{Discussion}

If the system (1) is discernable or $\mathcal{R}$-discernable, the whole active sequence $\sigma^{*}$ in the interval of time $[k-h, k]$ can be estimated at time $k$ with Proposition 10 or Proposition 14 respectively. Hence, the discernibility or the $\mathcal{R}$-discernibility allows to estimate at time $k=h$, the first active sequence $\sigma^{*}$ in the interval of time $[0, h]$ and so, all the modes $\sigma(k)$ for $k=0, \ldots, h$. By a receding horizon approach, they also allow to estimate at times $k>h$ the active sequence $\sigma^{*}$ in any interval of time $[k-h, k]$ and so all the modes $\sigma(k)$ for $k>h$ based on Corollaries 3 and 5. As a result, Proposition 10 or Proposition 14 together with Corollaries 3 and 5 allow to achieve the estimation, called permanent estimation, of all the modes $\sigma(k)$ for $k \geq 0$.

If the system (1) is $(\eta, \omega)$-discernable or $\mathcal{R}(\eta, \omega)$-discernable, the subsequence $\sigma_{[k-h+\eta, k-\omega]}^{*}$ of the active sequence $\sigma^{*}$ can be estimated at time $k$ by Proposition 16 or Proposition 17 respectively. Hence, the $(\eta, \omega)$-discernibility or the $\mathcal{R}(\eta, \omega)$-discernibility allows to estimate at time $k=h$, the first active subsequence $\sigma_{[\eta, h-\omega]}^{*}$ and then, all the modes $\sigma(k)$ for $k=\eta, \ldots, h-\omega$. The integers $\eta$ and $\omega$ should be as small as possible to get an estimated sequence of maximal length. However, the first part $\sigma_{[0, \eta-1]}^{*}$ of the active sequence cannot be estimated. By a receding horizon approach, they also allow to estimate at times $k>h$ the active subsequences $\sigma_{[k-h+\eta, k-\omega]}^{*}$ and so all the modes $\sigma(k)$ for $k>h-\omega$ based on Corollaries 6 and 7. As a result, Proposition 16 or Proposition 17 together with Corollaries 6 and 7 allow to achieve the estimation, called permanent estimation, of all the modes $\sigma(k)$ for $k \geq \eta$ up to a delay $\omega$.

The complexity of the estimation of the mode $\sigma(k)$ at time $k>h$, in terms of required amount of data and number of residuals to be calculated and analyzed, can be reduced. Indeed, the estimation of the mode $\sigma(k)$ at time $k$ can be performed taking into account that a finite numbers of successive modes at times $k^{\prime}<k$ have already been estimated and so are assumed to be known. In this respect, the notion of Backward Discernibility, introduced in [23], plays a central role as developed in next section. Then, we will propose an extension which will be useful in the case when the Backward Discernibility is not verified. It will be called the $(\lambda)$-Backward Discernibility. The property of $(\lambda)$-Backward Discernibility allows to estimate at time $k$, the mode $\sigma(k-\lambda)$, assuming that a finite numbers of successive modes at times $k^{\prime}<k-\lambda$ have already been estimated.

\section{BACKWARD DISCERNIBILITY AND RECURSIVE ESTIMATION}

\section{A. Backward Discernibility}

Let us denote with $\sigma^{h} i$ (resp. $\sigma^{h} j$ ) two modes sequences obtained from the concatenation of a sequence $\sigma^{h}$ of length $h$ and a mode $i \in$ $\mathcal{J}$ (resp. a mode $j \in \mathcal{J}$ ). More formally, the Backward Discernibility obeys the following definition.

Definition 9. [23] An active mode $i$ is Backward Discernable from another mode $j$ if there exists an integer $h$ such that for every sequence $\sigma^{h} \in \mathcal{J}^{h}$ of length $h, \sigma^{h} i$ is discernable from $\sigma^{h} j$. The smallest integer $h$ is called the Backward Discernibility index of $i$ from $j$.

In other words, the Backward Discernibility reflects the ability to discriminate between two sequences of same length in an interval of time $[k-h, k]$ which only differ by its last element $\sigma(k)$. We can introduce the notion of Backward Discernibility for the system (1).

Definition 10. The system (1) is Backward Discernable if, for all admissible sequences $\sigma^{h} i \in \mathcal{J}^{h+1}, \sigma^{h} j \in \mathcal{J}^{h+1}$ with $(i, j) \in \mathcal{J}^{2}$ 
and $\sigma^{h} \in \mathcal{J}^{h}$, the mode $i$ is Backward Discernable from the mode $j$.

Likewise for the discernibility, conditions for guaranteeing the Backward Discernibility can be stated.

Proposition 18. For a given input sequence $u_{k-h, k}$, an active mode $i$ is Backward Discernable from a mode $j$ on an observation window of length $h+1$, for almost all initial conditions $x_{k-h}$ iff, for every sequence $\sigma^{h} \in \mathcal{J}^{h}$ of length $h$ delivered by (1), at least one of the two following conditions is satisfied

$$
\begin{gathered}
\Omega_{\sigma^{h_{j}}} \mathcal{O}_{\sigma^{h_{i}}} \neq \mathbf{0} \\
\Omega_{\sigma^{h_{j}}}\left(\left(T_{\sigma^{h_{i}}}-T_{\sigma^{h_{j}}}\right) u_{k-h_{, k}}+\left(T_{\sigma^{h_{i}}}^{\prime}-T_{\sigma^{h_{j}}}^{\prime}\right)\right) \neq \mathbf{0}
\end{gathered}
$$

Proof. The proof is a straightforward consequence of the consideration of both Definition 9 of the Backward Discernibility and (29)-(30) of Theorem 3 .

When the Backward Discernibility is satisfied, the estimation of the mode $\sigma(k)$ at time $k$ can be performed according to the following proposition.

Proposition 19. Assume that (1) is Backward Discernable. Let $\sigma^{h}$ be a sequence of length $h$. Let $\mathcal{S}^{\sigma^{h}} \subset \mathcal{S}$ be the set of integers $s \in \mathcal{S}$ which uniquely identify the sequences $\sigma^{h} i$ for all $i \in \mathcal{J}$. The active mode $\sigma^{*}(k)$ in the interval of time $[k-h, k]$ is the unique mode $\sigma(k)$ of the sequence $\sigma_{s} \in \mathcal{J}^{h+1}\left(s \in \mathcal{S}^{\sigma^{h}}\right)$ such that the corresponding residual $r_{h, \sigma_{s}}$ is equal to zero.

Proof. Only the residuals of the restricted set $\mathcal{S}^{\sigma^{h}}$ of sequences sharing a common initial part of length $h$ are considered. The Backward Discernibility guarantees that only one residual in this set is equal to zero. It is necessarily the one which corresponds to the active mode $\sigma^{*}(k)$.

The Backward Discernibility allows to estimate the last mode of the active sequence, being assumed that the $h-1$ previous modes have already been estimated. The relevance of Proposition 19 lies in that only the residuals of the sequences $\sigma^{h} i(i \in \mathcal{J})$ with $\sigma^{h} \in \mathcal{J}^{h}$ resulting from the estimation at time $k-1$ have to be checked by the detector. In some cases, that allows to drastically reduce the number of tests: $J$ residuals to be tested instead of $J^{h+1}$ residuals.

An equivalent condition of (48) that holds regardless of the structure and does not require the computation of $\Omega_{\sigma^{h}}$ can be proposed.

Proposition 20. Condition (48) is equivalent to

$$
\operatorname{rank}\left(\left[\begin{array}{ll}
\mathcal{O}_{\sigma^{h_{i}}} & \mathcal{O}_{\sigma^{h}}
\end{array}\right]\right)>\operatorname{rank}\left(\mathcal{O}_{\sigma^{h_{j}}}\right)
$$

Proof. The proof is a straightforward consequence of the consideration of both Definition 9 of the Backward Discernibility and Proposition 11 replacing $\sigma_{s_{i}}$ by $\sigma^{h} i$ and $\sigma_{s_{j}}$ by $\sigma^{h} j$.

An equivalent condition of (50) is proposed here and will be useful to decide on the Backward Discernibility of (1) by examining the property of the matrices $A_{\sigma(k)}$.

Proposition 21. Assume that there exists an integer $h \in \mathbb{N}$ such that (27) is satisfied. A mode $i$ is Backward Discernable from a mode $j$ if

$$
\left(C_{i}-C_{j}\right) A_{\sigma(k-1)}^{\sigma(k-h)} \neq \mathbf{0}
$$

Proof. Assume that there exists an integer $h \in \mathbb{N}$ such that (27) is satisfied. Taking $\sigma_{[k-h, k]}=\sigma^{h} j$, (27) is equivalent to

$$
\operatorname{rank}\left(\mathcal{O}_{\sigma^{h}}\right)=\operatorname{rank}\left(\mathcal{O}_{\sigma^{h}}\right) \quad \forall j \in \mathcal{J}
$$

Hence, (50) turns into

$$
\operatorname{rank}\left(\left[\begin{array}{ll}
\mathcal{O}_{\sigma^{h}} & \mathcal{O}_{\sigma^{h}}
\end{array}\right]\right)>\operatorname{rank}\left(\mathcal{O}_{\sigma^{h}}\right)
$$

Let us denote with $V_{h+1}^{i}, V_{h+1}^{j}$ the last rows of $\mathcal{O}_{\sigma^{h}}$ and $\mathcal{O}_{\sigma^{h}}$ respectively.

Since the rows of $\mathcal{O}_{\sigma^{h}}$ coincide with the rows of $\mathcal{O}_{\sigma^{h}}$ except the last one, (52) is satisfied if the row $\left[\begin{array}{ll}V_{h+1}^{i} & V_{h+1}^{j}\end{array}\right]$ is not a linear combination of the other rows of $\left[\begin{array}{ll}\mathcal{O}_{\sigma^{h}} & \mathcal{O}_{\sigma^{h}}\end{array}\right]$, which means that there exists a h-uple $\left(\alpha_{1}, \ldots, \alpha_{h}\right) \in \mathbb{R}^{h}$ such that

$$
\left[\begin{array}{ll}
V_{h+1}^{i} & V_{h+1}^{j}
\end{array}\right] \neq \alpha_{1}\left[\begin{array}{ll}
V_{1} & V_{1}
\end{array}\right]+\alpha_{2}\left[\begin{array}{ll}
V_{2} & V_{2}
\end{array}\right]+\cdots+\alpha_{h}\left[\begin{array}{ll}
V_{h} & V_{h}
\end{array}\right]
$$

where $V_{l}(l=1, \ldots, h)$ denotes the $i^{\text {th }}$ row of $\mathcal{O}_{\sigma^{h}}$. This is equivalent to

$$
\left[\begin{array}{ll}
V_{h+1}^{i} & V_{h+1}^{j}
\end{array}\right] \neq\left[\alpha_{1} V_{1}+\cdots+\alpha_{h} V_{h} \quad \alpha_{1} V_{1}+\cdots+\alpha_{h} V_{h}\right]
$$

and so to

$$
V_{h+1}^{i} \neq V_{h+1}^{j}
$$

Since

$$
V_{h+1}^{i}=C_{i} A_{\sigma(k-1)}^{\sigma(k-h)} \text { and } V_{h+1}^{j}=C_{j} A_{\sigma(k-1)}^{\sigma(k-h)}
$$

we get that

$$
V_{h+1}^{i} \neq V_{h+1}^{j} \Leftrightarrow\left(C_{i}-C_{j}\right) A_{\sigma(k-1)}^{\sigma(k-h)} \neq \mathbf{0}
$$

which is nothing but (51). That completes the proof.

Remark 5. If $C_{\sigma(k)}=C$, that is if the output matrix does not depend on the mode, (51) turns into

$$
C\left(A_{i}-A_{j}\right) A_{\sigma(k-2)}^{\sigma(k-h)} \neq \mathbf{0}
$$

Remark 6. The equation (51) means that if the product $A_{\sigma(k-1)}^{\sigma(k-h)}$ is not invertible, the Backward Discernibility of $i$ from $j$ is ensured if $\left(C_{i}-C_{j}\right)^{T}$ does not belong to the kernel of $A_{\sigma(k-1)}^{\sigma(k-h)^{T}}$. Furthermore, if all the matrices $A_{\sigma(k-1)} \cdots A_{\sigma(k-h)}$ are full rank, then $A_{\sigma(k-1)}^{\sigma(k-h)}$ is also full rank. Hence, the kernel of the product $A_{\sigma(k-1)}^{\sigma(k-h)^{T}}$ reduces to zero. As a result, $\left(C_{i}-C_{j}\right) A_{\sigma(k-1)}^{\sigma(k-h)}=0$ holds whenever $C_{i}=C_{j}$.

Based on Proposition 21, we can easily state the following property of reversibility which, unlike the discernibility, applies to the Backward Discernibility.

Corollary 8. Assume that there exists an integer $h \in \mathbb{N}$ such that (27) is satisfied. Consider two modes $i \in \mathcal{J}$ and $j \in \mathcal{J}$ with $j \neq i$. The two following statements A3 et A4 are equivalent

A3) the mode $i$ is Backward Discernable from the mode $j$ A4) the mode $j$ is Backward Discernable from the mode $i$ It is said that the Backward Discernibility is reversible.

Proof. From Proposition 21, a mode $i$ is Backward Discernable from another mode $j$ if

$$
\left(C_{i}-C_{j}\right) A_{\sigma(k-1)}^{\sigma(k-h)} \neq \mathbf{0}
$$

On the other hand, a mode $j$ is Backward Discernable from another mode $i$ if

$$
\left(C_{j}-C_{i}\right) A_{\sigma(k-1)}^{\sigma(k-h)} \neq \mathbf{0}
$$

Clearly, (55) is equivalent to (56). It means that the Backward Discernibility is reversible.

As a straightforward but useful extension, a less restricted case can also be considered. We call it here the $(\lambda)$-Backward Discernibility. 


\section{B. ( $\lambda)$-Backward Discernibility}

The property of $(\lambda)$-Backward Discernibility allows to discriminate at time $k$, the mode $\sigma(k-\lambda)$ from all the other modes, being the modes $\sigma(k-i)(1+\lambda \leq i \leq h)$ known. The quantity $\lambda$ is an integer such that $\lambda \in\{0, \ldots, h\}$. The case when $\lambda=0$ amounts to merely consider the Backward Discernibility while $\lambda=h$ corresponds to the $(\eta=0, \omega=h)$-Discernibility. More formally, the $(\lambda)$-Backward Discernibility obeys the following definition.

Definition 11. An active mode $i$ is ( $\lambda$-Backward Discernable from another mode $j$, if there exists an integer $\lambda$ such that, for every sequence $\sigma^{h-\lambda} \in \mathcal{J}^{h-\lambda}$ of length $h-\lambda$, and for any two sequences $\sigma_{1}^{\prime}$ and $\sigma_{2}^{\prime}$ of length $\lambda, \sigma^{h-\lambda} i \sigma_{1}^{\prime}$ is discernable from $\sigma^{h-\lambda} j \sigma_{2}^{\prime}$.

We can introduce the notion of $(\lambda)$-Backward Discernibility for the system (1).

Definition 12. The system (1) is ( $\lambda)$-Backward Discernable if, for every pairs of modes $(i, j) \in \mathcal{J}^{2}$, the mode $i$ is $(\lambda)$-Backward Discernable from the mode $j$.

Conditions for guaranteeing the $(\lambda)$-Backward Discernibility can be expressed in a similar way as for the Backward Discernibility.

Proposition 22. For a given input sequence $u_{k-h, k}$, an active mode $i$ is $(\lambda)$-Backward Discernable from a mode $j$ on an observation window of length $h+1$, for almost all initial conditions $x_{k-h}$ iff, for every sequence $\sigma^{h-\lambda} \in \mathcal{J}^{h-\lambda}$ of length $h-\lambda$ and for any two sequences $\sigma_{1}^{\prime}$ and $\sigma_{2}^{\prime}$ of length $\lambda$ delivered by (1), at least one of the following conditions are satisfied

$$
\begin{gathered}
\Omega_{\sigma^{h-\lambda} j \sigma_{1}^{\prime}} \mathcal{O}_{\sigma^{h-\lambda} i \sigma_{2}^{\prime}} \neq \mathbf{0} \\
\Omega_{\sigma^{h-\lambda} j \sigma_{1}^{\prime}}\left(\left(T_{\sigma^{h-\lambda} i \sigma_{2}^{\prime}}-T_{\sigma^{h-\lambda} j \sigma_{1}^{\prime}}\right) u_{k-h, k}\right. \\
\left.+\left(T_{\sigma^{h-\lambda} i \sigma_{2}^{\prime}}^{\prime}-T_{\sigma^{h-\lambda} j \sigma_{1}^{\prime}}^{\prime}\right)\right) \neq \mathbf{0}
\end{gathered}
$$

Proof. The proof is a straightforward consequence of the consideration of both Definition 11 of the $(\lambda)$-Backward Discernibility and (29)-(30) of Theorem 3.

When the $(\lambda)$-Backward Discernibility is satisfied, the estimation of the mode $\sigma(k-\lambda)$ at time $k$ can be performed according to the following proposition.

Proposition 23. Assume that (1) is $(\lambda)$-Backward Discernable. Let $\sigma^{h-\lambda}$ be a sequence of length $h-\lambda$. Let $\mathcal{S}^{\sigma^{h-\lambda}} \subset \mathcal{S}$ be the set of integers $s \in \mathcal{S}$ which uniquely identify the sequences $\sigma^{h-\lambda} i \sigma^{\prime}$ for all $i \in \mathcal{J}$ and for all sequences $\sigma^{\prime} \in \mathcal{J}^{\lambda}$ of length $\lambda$. The active mode $\sigma^{*}(k-\lambda)$ in the interval of time $[k-h, k]$ is the unique mode $\sigma(k-\lambda)$ of the sequence $\sigma_{s} \in \mathcal{J}^{h+1}\left(s \in \mathcal{S}^{\sigma^{h-\lambda}}\right)$ such that the corresponding residual $r_{h, \sigma_{s}}$ is equal to zero.

Proof. Only the residuals $r_{h, \sigma_{s}}$ of the sequences $\sigma^{h-\lambda} i \sigma^{\prime}$ for all $i \in \mathcal{J}$ and all sequences $\sigma^{\prime} \in \mathcal{J}^{\lambda}$ of length $\lambda$ are considered. The $(\lambda)$-Backward Discernibility guarantees that only one residual among these sequences is null. It is necessarily the one corresponding to the active mode.

The $(\lambda)$-Backward Discernibility allows to estimate a specific mode in a sequence, being assumed that the $h-\lambda-1$ previous modes have already been estimated. The relevance of Proposition 23 lies in that only the residuals of the sequences $\sigma^{h-\lambda} i \sigma^{\prime}\left(\sigma^{\prime} \in \mathcal{J}^{\lambda}\right)$ with $\sigma^{h-\lambda} \in \mathcal{J}^{h-\lambda}$ resulting from the estimation at time $k-1$ have to be checked by the detector. That allows to reduce the number of tests: $J^{\lambda+1}$ sequences instead of $J^{h+1}$ sequences (let us recall that $\lambda \leq h)$. Let us note that there is a delay equal to $\lambda$ between the time instants of the estimation and of the active mode. It is clear that the smaller $\lambda$, the shorter the delay.

\section{OVERALL MODES DETECTION PROCEDURE}

This section aims at giving, by recapitulating the previous results, a detailed description of the overall procedure needed for the modes detections of (1). The procedure is splitted into two parts: the estimation of the first sequence called initial estimation. It is followed by the estimation of the remaining sequence, called permanent estimation. The modes detection is performed from the knowledge of the input/output data on an observation window of length $h+1$ either with a receding horizon technique or a recursive estimation. In both cases, the permanent estimation is performed on-line.

Remark 7. Let us point out that if (1) is $(\eta, \omega)$-discernable, it may exist integers $\eta^{\prime}<\eta$ and/or $\omega^{\prime}<\omega$ such that (1) is $\mathcal{R}\left(\eta^{\prime}, \omega^{\prime}\right)$ discernable, and in such a case, a larger sequence can be estimated.

The overall procedure is given in Figure 1 and detailed below.

\section{Off-line steps}

Step 1: detection horizon $h$ Calculate the minimal integer $h$ such that (27) is satisfied. Calculate the matrices $\Omega_{s}$ as the solutions of (10).

Step 2: discernibility Check whether conditions (29) and (30) of Theorem 3 are satisfied. If the discernibility is verified, go to Step 6 . Otherwise, go to the next step.

Step 3: $\mathcal{R}$-discernibility Check whether (40) is fulfilled. If the $\mathcal{R}$ discernibility is verified, go to Step 6. Otherwise, go to the next step. Step 4: $(\eta, \omega)$-discernibility Calculate $\eta$ and $\omega$ such that (42) or (43) of Proposition 15 is fulfilled. The integers are initialized to $\eta=0$ and $\omega=1$ (let us recall that $\omega=0$ corresponds to the discernibility as reported in Figure 1), then increased until (42) or (43) is satisfied. Go to the next step.

Step 5: $\mathcal{R}(\eta, \omega)$-discernibility Calculate $\eta$ and $\omega$ such that (45) is fulfilled. The integers are initialized to $\eta=0$ and $\omega=1$ (let us recall that $\omega=0$ corresponds to the $\mathcal{R}$-discernibility as reported in Figure 1), then increased until (45) is satisfied. Go to the next step. If there aren't any $\eta$ and $\omega$, the modes detection cannot be achieved. Step 6: Backward Discernibility

- if the discernibility (from Step 2) is satisfied, the Backward Discernibility is necessarily satisfied according to the second inference reported in Figure 1.

- if the $\mathcal{R}$-discernibility (from Step 3 ) or if the $(\eta, \omega)$-discernibility (from Step 4) or if the $\mathcal{R}(\eta, \omega)$-discernibility (from Step 5) is satisfied, the Backward Discernibility has to be tested. The Backward Discernibility is tested with (48) (or alternatively with (51)) and (49) of Proposition 18. If Backward Discernibility is satisfied, go to Step 8. Otherwise, go to the next step.

Step 7: $(\lambda)$-Backward Discernibility Check whether (57) or (58) of Proposition 22 is satisfied. The integer $\lambda$ is initialized to $\lambda=1$ (let us recall that $\lambda=0$ corresponds to the Backward Discernibility as reported in Figure 1), then increased until (57) or (58) is satisfied. Go to the next step.

\section{On-line steps}

\section{Step 8: initial estimation}

- If the system (1) is discernable or $\mathcal{R}$-discernable, at time $k=h$, the active sequence $\sigma^{*}$ in the interval of time $[0, h]$ is estimated by (28) of Proposition 10 or by (41) of Proposition 14 respectively and go to Step 9. Otherwise, go to the next step.

- If the system (1) is $(\eta, \omega)$-discernable or $\mathcal{R}(\eta, \omega)$-discernable, at time $k=h$, the subsequence $\sigma_{[\eta, h-\omega]}^{*}$ of the active sequence $\sigma^{*}$ is estimated by (44) of Proposition 16 or by (47) of Proposition 17 accordingly. Then, increase $k$ and use (44) or (47) until a sequence of length $h$ or $h-\lambda$ is known according to the consideration of the Backward Discernibility or the $(\lambda)$-Backward Discernibility.

Step 9: permanent estimation 
- If the Backward Discernibility or the $(\lambda)$-Backward Discernibility is satisfied, the permanent estimation can be performed in a recursive way from $k$ to $k+1$ by resorting to Proposition 19 or to Proposition 23 respectively.

- If neither the Backward Discernibility nor the $(\lambda)$-Backward Discernibility are satisfied, refer to either Proposition 10 together with Corollary 3 or Proposition 14 together with Corollary 5 or Proposition 16 together with Corollary 6 or Proposition 17 together with Corollary 7 through a receding horizon technique.

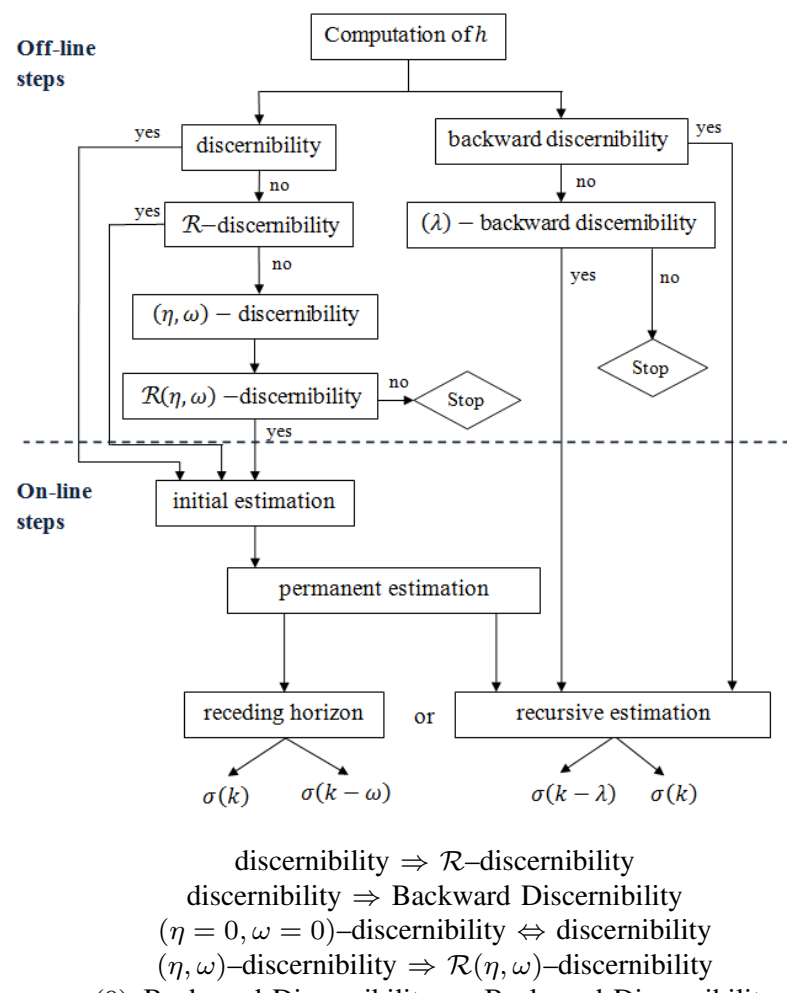

(0)-Backward Discernibility $\Leftrightarrow$ Backward Discernibility

(h)-Backward Discernibility $\Leftrightarrow(\eta=0, \omega=h)$-discernibility

Forward Discernibility $\Leftrightarrow(\eta=0, \omega)$-discernibility

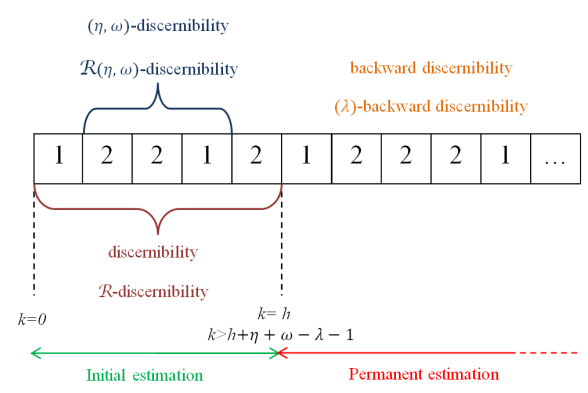

Fig. 1. Top: Overall modes detection procedure. Middle: relations between the distinct notions of discernibility. Bottom: illustration of modes detection on a sequence of modes 1 or 2

\section{EXAMPLE}

Consider the switched system of the form (1) with

$$
\begin{gathered}
A_{1}=\left[\begin{array}{ll}
1 & 0 \\
1 & 0
\end{array}\right], \quad A_{2}=\left[\begin{array}{cc}
1 & -0.5 \\
1 & 0
\end{array}\right], \quad C_{1}=C_{2}=\left[\begin{array}{ll}
2 & 0
\end{array}\right] \\
B_{1}=B_{2}=\left[\begin{array}{l}
0 \\
0
\end{array}\right], \quad D_{1}=D_{2}=0, \quad E_{1}=E_{2}=\left[\begin{array}{l}
1 \\
0
\end{array}\right]
\end{gathered}
$$

\section{Off-line steps}

Step 1: detection horizon The smallest detection horizon $h$ which fulfills (27) is $h=2$.

Step 2: discernibility Since $B_{1}=B_{2}=\mathbf{0}, E_{1}=E_{2}$ and $C_{1}=C_{2}$, (30) is never fulfilled according to Remark 4. Hence, only the test (29), or alternatively the test (31) must be performed. The result is reported in Figure 2. For every sequences $\sigma_{s} \in \mathcal{J}^{h+1=3}$ $(s \in \mathcal{S})$, the corresponding non discernable sequences of the same length, if any, are reported on the same row. From Figure 2, it is

\begin{tabular}{|c|cccc|}
\hline Seq. $\sigma_{s_{i}}$ & Seq. $\sigma_{s_{j}}$ from & which $\sigma_{s_{i}}$ is not discernable \\
\hline 111 & 111 & 112 & 211 & 212 \\
\hline 112 & 112 & 111 & 211 & 212 \\
\hline 121 & 121 & 122 & 221 & 222 \\
\hline 122 & 122 & 121 & 221 & 222 \\
\hline 211 & & 211 & 212 & \\
\hline 212 & & 212 & 211 & \\
\hline 221 & 221 & 222 & \\
\hline 222 & & 222 & 221 & \\
\hline
\end{tabular}
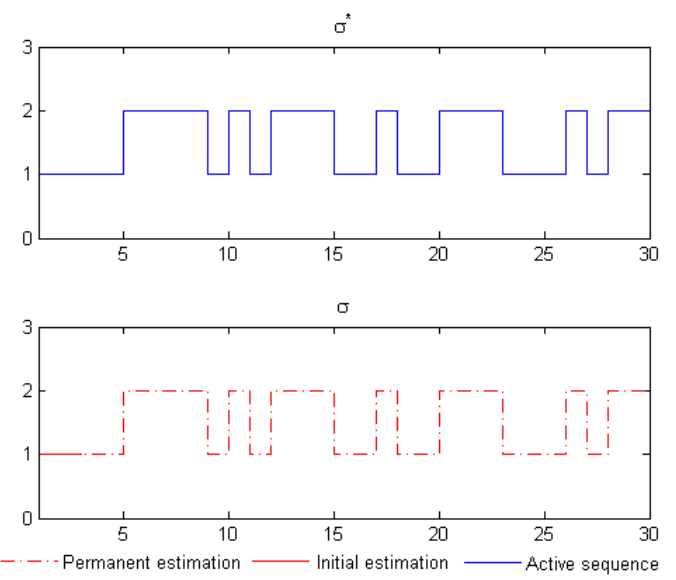

Fig. 2. Top: non discernable sequences. Middle: Active sequence $\sigma^{*}$ Bottom: reconstructed sequence $\sigma$

clear that there are not any discernable sequences. Thus, the system is not discernable.

Step 3: $\mathcal{R}$-discernibility Since $B_{1}=B_{2}=\mathbf{0}, E_{1}=E_{2}$ and $C_{1}=C_{2}$, the sets $\mathcal{R}_{\sigma_{i}}(i=1, \ldots, 8)$ are built from (38). They are $\mathcal{R}_{\sigma_{1}}=\{111,112,211,212\}, \mathcal{R}_{\sigma_{2}}=\{111,112,211,212\}, \mathcal{R}_{\sigma_{3}}=$ $\{121,122,221,222\}, \mathcal{R}_{\sigma_{4}}=\{121,122,221,222\}, \mathcal{R}_{\sigma_{5}}=$ $\{211,212\}, \mathcal{R}_{\sigma_{6}}=\{211,212\}, \mathcal{R}_{\sigma_{7}}=\{221,222\}, \mathcal{R}_{\sigma_{8}}=$ $\{221,222\}$. It holds that (40) is not satisfied for all $\left(s_{i}, s_{j}\right) \in$ $\{1, \cdots, 8\} \times\{1, \cdots, 8\}$ since $\mathcal{R}_{\sigma_{1}}=\mathcal{R}_{\sigma_{2}}, \mathcal{R}_{\sigma_{3}}=\mathcal{R}_{\sigma_{4}}, \mathcal{R}_{\sigma_{5}}=$ $\mathcal{R}_{\sigma_{6}}$ and $\mathcal{R}_{\sigma_{7}}=\mathcal{R}_{\sigma_{8}}$. Consequently, according to Definition 4 , the system is not $\mathcal{R}$-discernable.

Step 4: $(\eta, \omega)$-discernibility The property of $(\eta, \omega)$-discernibility is checked using (42) since (43) is never verified because $B_{1}=B_{2}=\mathbf{0}$, $E_{1}=E_{2}$ and $C_{1}=C_{2}$. From Figure 2, it holds that (42) is satisfied for $\eta=1$ and $\omega=1$. Hence, the $(1,1)$-discernibility is fulfilled.

Step 5: $\mathcal{R}(\eta, \omega)$-discernibility The property of $\mathcal{R}(\eta, \omega)-$ discernibility is checked using (45). It holds that (45) is satisfied with $\eta=0$ and $\omega=1$, that is $\mathcal{R}(0,1)$-discernibility is fulfilled.

It is an illustration of what was stressed in Remark 7. Indeed, if the $(\eta, \omega)$-discernibility is verified, it was pointed out that we might find $\eta^{\prime} \leq \eta$ and/or $\omega^{\prime} \leq \omega$ such that the $\mathcal{R}\left(\eta^{\prime}, \omega^{\prime}\right)$-discernibility is verified. In this example, $\eta^{\prime}=0<\eta=1$ and $\omega^{\prime}=\omega=1$.

Step 6: Backward Discernibility Since $B_{1}=B_{2}=\mathbf{0}, E_{1}=E_{2}$ and $C_{1}=C_{2}$, the Backward Discernibility has to be tested with only (48) of Proposition 18 or equivalently (51). It holds that the Backward Discernibility is not satisfied. 
Step 7: $(\lambda)$-Backward Discernibility Since $B_{1}=B_{2}=\mathbf{0}$, $E_{1}=E_{2}$ and $C_{1}=C_{2}$, the $(\lambda)$-Backward Discernibility has to be tested with only condition (57) of Proposition 22. It holds that (57) is fulfilled for $\lambda=1$.

On-line steps

Step 8: initial estimation Since the $\mathcal{R}(0,1)$-discernibility is verified, at time $k=2$, the subsequence $\sigma_{[0,1]}^{*}$ of the active sequence $\sigma^{*}$ is estimated by (47) of Proposition 17.

Step 9: permanent estimation Since the (1)-Backward Discernibility is satisfied, the permanent estimation is performed by resorting to Proposition 23. The detector delivers the active mode $\sigma^{*}(k-1)$ for $k>h+\omega+\eta-\lambda-1=1$ by testing 2 residuals among the $2^{3}=8$ residuals. The detector successfully recovers the unknown mode as depicted on Figure 2.

\section{CONCLUSION}

We have addressed the problem of modes detection for switching discrete-time linear and affine systems. A unified presentation of the main model-based modes detection methods proposed in the literature has been carried out and the methods have been compared. The central notion of discernibility has been thoroughly discussed. A complete step-by-step procedure has been detailed for the detection of the modes. We hope that this tutorial will be helpful for people working in various realms like observer synthesis, gain scheduling, hybrid systems or diagnosis.

\section{REFERENCES}

[1] A.j. Juloski, W.P.M.H. Heemels, and G. Ferrari-Trecate. Identification of an experimental hybrid system. In IFAC ADHS, pages 46-51, St Malo, France, 2003.

[2] Lala H. Rajaoarisoa, Nacer K. M'Sirdi, and J-F Balmat. Micro-climate optimal control for an experimental greenhouse automation. In Proc. of IEEE International Conference on Communications, Computing and Control Applications, Marseille, France, 2012.

[3] M. Yua, D. Wanga, M. Luob, D. Zhangb, and Q. Chenc. Fault detection, isolation and identification for hybrid systems with unknown mode changes and fault patterns. Expert Systems with Applications, 39(11):9955-9965, 2012.

[4] A. Alessandri and P. Coletta. Design of luenberger observers for a class of hybrid linear systems. In M. D. Di Benedetto and A. SangiovanniVincentelli, editors, Hybrid Systems: Computation and Control, volume 2034 of $L N C S$, pages 7-18. Springer, 2001.

[5] A. Alessandri and P. Coletta. Switching observers for continuous-time and discrete-time linear systems. In Proc. of IEEE ACC, 2001.

[6] J. Daafouz, P. Riedinger, and C. Iung. Stability analysis and control synthesis for switched systems : A switched lyapunov approach. IEEE Transactions on Automatic Control, November 2002.

[7] A. Lj. Juloski, W. P. M. H. Heemels, and S. Weiland. Observer design for a class of piecewise linear systems. International Journal of Robust and Nonlinear Control, pages 1387-1404, 2007.

[8] R. Vidal. Identification of pwarx hybrid models with unknown and possibly different orders. In In Proc. of IEEE ACC, 2004.

[9] G. Ferrari-Trecate, M.Muselli, D. Liberati, and M. Morari. A clustering technique for the identification of piecewise affine systems. Automatica, 39(2):205-217, 2003.

[10] A. Bemporad, A. Garulli, S. Paoletti, and A. Vicino. A bounded-error approach to piecewise affine system identification. IEEE Trans. on Automatic Control, 50(10):1567-1580, 2005.

[11] L. Bako. Identification of switched linear systems via sparse optimization. Automatica, 47(4):668-677, 2011.

[12] H. Ohlsson and L. Ljung. Identification of switched linear regression models using sum-of-norms regularization. Automatica, 49(4), 2013.

[13] J. Borges, V. Verdult, M. Verhaegen, and M. A. Botto. A switching detection method based on projected subspace classification. In In Conference on Decision and Control-European Control Conference, Seville, Spain, 2005.

[14] K. M. Pekpe, G. Mourot, K. Gasso, and J. Ragot. Identification of switching systems using change detection technique in the subspace framework. In In Conference on Decision and Control, Atlantis, Paradise Island, Bahamas, 2004.
[15] K. Huang, A. Wagner, and Y. Ma. Identification of hybrid linear timeinvariant systems via subspace embedding and segmentation. In Proc. of 43rd conference on Decision and Control (CDC 2004), Bahamas, December 2004.

[16] A. Balluchi, L. Benvenuti, M. D. Di Benedetto, and A. L. SangiovanniVincentelli. Design of Observers for Hybrid Systems. In In Proceeding of the 5th International Workshop on Hybrid Systems, volume 2289, pages 76-89, Stanford, Calif, USA, 2002.

[17] A. Jaluski, M. Heemels, and S. Weiland. Observer design for a class of piecewise affine systems. In Proc. of 41st conference on Decision and Control (CDC 2002), Las Vegas, 2002.

[18] P. M. Frank. Fault diagnosis in dynamic systems using analytical and knowledge-based redundancy: A survey and some new results. Automatica, 26(3):459-474, 1990.

[19] V. Venkatasubramanian, R. Rengaswamy, K. Yin, and S. N. Kavuri. A review of process fault detection and diagnosis: Part I: Quantitative modelbased methods. Computers and Chemical Engineering, 27(3):293-311, 2003.

[20] A. Alessandri, M. Baglietto, and G. Battistelli. Receding-horizon estimation for switching discrete-time linear systems. IEEE Transactions on Automatic Control, 50(11), 2005.

[21] J. Prakash, S.C. Patwardhan, and S.L. Shah. State estimation and nonlinear predictive control of autonomous hybrid system using derivative free state estimators. Journal of Process Control, 20(7):787-799, 2010.

[22] M. Fliess, C. Join, and W. Perruquetti. Real-time estimation for switched linear system. In Proc. of 47th IEEE Conference on Decision and Control (CDC'2008), Cancun, Mexico, December 2008.

[23] M. Babaali and M. Egerstedt. Asymptotic observers for discrete-time switched linear systems. In In Proceeding of the 16th IFAC World Congress, Czech Republic, 2005.

[24] E. A. Domlan, J. Ragot, and D. Maquin. Switching systems mode estimation using a model-based diagnosis method. In In 8th Conference on Diagnostics of Processes and Systems, Slubice, Poland, 2007.

[25] R. Vidal, A. Chiuso, and S. Soatto. Observability and identifiability of jump linear systems. In In Proceeding of IEEE Conference on Decision and Control, 2002.

[26] M. Baglietto, G. Battistelli, H. V. Hultmann Ayala, and P. Tesi. Modeobservability conditions for linear and nonlinear systems. In Proc. of 51 st IEEE Conference on Decision and Control CDC'2012, Maui, Hawaii, December 2012

[27] M. Baglietto, G. Battistelli, and P. Tesi. Projection-based degree of distinguishability in switching linear systems. In Proc. of 52nd IEEE Conference on Decision and Control CDC'2013, Florence, Italy, December 2013.

[28] M. Baglietto, G. Battistelli, and P. Tesi. Mode-observability degree in discrete-time switching linear systems. Systems \& Control Letters, 70(0):69 - 76, 2014.

[29] M. Babaali and M. Egerstedt. Observability of switched linear systems. Hybrids Systems: Computation and Control, pages 48-63, 2004.

[30] M. Baglietto, G. Battistelli, and L. Scardovi. Active mode observability of switching linear systems. Automatica, 43(11):1442-1449, 2007.

[31] M. Baglietto, G. Battistelli, and L. Scardovi. Active mode observation of switching linear systems based on set-valued estimation on the continuous state. International Journal Robust Nonlinear Control, 19:1521-1540, 2009.

[32] M. Babaali and M. Egerstedt. Pathwise observability and controllability are decidable. In Proceedings of the 42nd IEEE conference on decision and control (CDC'03), volume 6, pages 5771-5776, Maui, Hawaii, December 2003.

[33] S. Paoletti, A. Garulli, J. Roll, and A. Vicino. A necessary and sufficient condition for Input realization of switched affine state space models. In In Proceeding of the 47th IEEE conference on decision and control, Cancun, Mexico, December 2008.

[34] J. C. A. Barata and M. S. Hussein. The Moore-Penrose Pseudoinverse: A Tutorial Review of the Theory. Brazilian Journal of Physics, 42:146$165,2012$. 